\title{
The Long-Lived Disks in the $\eta$ Chamaeleontis Cluster
}

\author{
Aurora Sicilia-Aguilar ${ }^{1}$, Jeroen Bouwman ${ }^{1}$, Attila Juhász ${ }^{1}$, Thomas Henning ${ }^{1}$, \\ Veronica Roccatagliata ${ }^{1}$, Warrick A. Lawson ${ }^{2}$, Bram Acke $^{3,4}$, \\ Eric D. Feigelson ${ }^{5}$, A.G.G.M. Tielens ${ }^{6}$, Leen Decin ${ }^{3}$, Gwendolyn Meeus $^{7}$ \\ sicilia@mpia.de
}

\begin{abstract}
We present IRS spectra and revised MIPS photometry for the 18 members of the $\eta$ Chamaeleontis cluster. Aged $8 \mathrm{Myr}$, the $\eta$ Cha cluster is one of the few nearby regions within the 5-10 Myr age range, during which the disk fraction decreases dramatically and giant planet formation must come to an end. For the 15 low-mass members, we measure a disk fraction $\sim 50 \%$, high for their $8 \mathrm{Myr}$ age, and 4 of the 8 disks lack near-IR excesses, consistent with the empirical definition of "transition" disks. Most of the disks are comparable to geometrically flat disks. The comparison with regions of different ages suggests that at least some of the "transition" disks may represent the normal type of disk around low-mass stars. Therefore, their flattened structure and inner holes may be related to other factors (initial masses of the disk and the star, environment, binarity), rather than to pure time evolution. We analyze the silicate dust in the disk atmosphere, finding moderate crystalline fractions $(\sim 10-30 \%)$ and typical grain sizes $\sim 1-3 \mu \mathrm{m}$, without any characteristic trend in the composition. These results are common to other regions of different ages, suggesting that the initial grain processing occurs very early in the disk lifetime $(<1 \mathrm{Myr})$. Large grain sizes in the disk atmosphere cannot be used as a proxy for age, but are likely related to higher disk turbulence. The dust mineralogy varies between the 8-12 $\mu \mathrm{m}$ and the 20-30 $\mu \mathrm{m}$ features, suggesting high temperature dust processing and little radial mixing. Finally, the analysis of IR and optical data on the B9 star $\eta$ Cha reveals that it is probably surrounded by a young debris disk with a large inner hole, instead of being a classical Be star.
\end{abstract}

\footnotetext{
${ }^{1}$ Max-Planck-Institut für Astronomie, Königstuhl 17, 69117 Heidelberg, Germany

${ }^{2}$ School of Physical, Environmental, and Mathematical Sciences, University of New South Wales, Australian Defense Force Academy, Canberra ACT 2600, Australia

${ }^{3}$ Instituut voor Sterrenkunde, KU Leuven, Celestijnenlaan 200D, 3001 Leuven, Belgium

${ }^{4}$ Postdoctoral Fellow of the Fund for Scientific Research, Flanders.

${ }^{5}$ Department of Astronomy and Astrophysics, Pennsylvania State University, University Park, PA 16802

${ }^{6}$ Kapteyn Astronomical Institute, PO Box 800, 9700 AU Groningen, The Netherlands

${ }^{7}$ Astrophysical Institute Potsdam (AIP), An der Sternwarte 16, 14482 Potsdam, Germany
} 
Subject headings: accretion disks — planetary systems: protoplanetary disks — stars: pre-main sequence

\section{Introduction}

The $\eta$ Chamaeleontis cluster is the nearest cluster discovered in the 20th century, and the first open cluster discovered by X ray observations (Mamajek et al. 1999). Located at $97 \mathrm{pc}$ distance, and associated to the B9 star $\eta$ Cha, its age has been estimated to be 5-9 Myr (Mamajek et al. 1999; Lawson et al. 2001; Luhman \& Steeghs 2004), falling within the most interesting and less studied epoch, when most disks disperse and giant planet formation has to conclude. The $\eta$ Cha cluster is moreover a remarkable star-forming region. Its location out of the galactic plane and far from molecular clouds raised questions about its origin, with the clusters space motions suggesting that it is probably related to the Sco-Cen star-forming clouds (Mamajek et al. 2000; De Zeeuw et al. 1999). The initial mass function (IMF) of the $\eta$ Cha cluster is unusual. Assuming an IMF consistent with rich young clusters and field stars, Lyo et al. (2004a) predicted 20-30 cluster members with masses under $0.15 \mathrm{M}_{\odot}$, but deep photometric surveys of large areas of the cluster that should have detected brown dwarfs (BD) and planetary-mass objects revealed no very low-mass members (Luhman 2004a; Lyo et al. 2006). This suggested either an abnormal IMF or dynamical ejection of the very low-mass objects during the early phases of formation of the cluster (Lawson et al. 2001; Moraux et al. 2007).

The $\eta$ Cha cluster has been one of the most interesting targets for Spitzer, given its close distance, which enables its members to be well characterized in optical properties, spectral type, and binarity. Although well-accepted evidence from other star forming regions suggested that most IR excesses from protoplanetary disks have disappeared by 6 My (Haisch et al. 2001), the $\eta$ Cha cluster appeared to have more accreting objects than expected from ground-based JHKL photometry (Haisch et al. 2005). The presence of a relatively large number of disks (disk fraction $\sim 50 \%$ ) was later confirmed with Spitzer IRAC photometry by Megeath et al. (2005). The number of "transition" objects (TO) or objects with inner opacity holes (no or very small near-IR excess emission, having therefore evacuated or optically thin disks at these wavelengths) was larger than observed in Taurus or in massive star-forming regions (Megeath et al. 2005), explaining the initial disagreement between near-IR excesses and accretion. Bouwman et al. (2006) used the IRS spectra of the low-mass members to determine the disk fraction for single and multiple stars, revealing that the lifetimes of disks in close-in binary systems are significantly shorter than the lifetimes of disks around single stars ( $\sim 5$ versus $\sim 9$ Myr, respectively). The high disk fraction in the cluster is linked to the single stars, as all but one of the binaries (the widest one, RECX-9) lack disks. They also found that the angular momentum of the star is related to the presence of disks and companions: the stars with disks are slow rotators, and the fastest rotators are in binary systems. This is consistent with the disk locking hypothesis, as binaries would have had more time to spin up after losing their disks (Bouwman et al. 2006). 
Here we study the disk properties of the cluster members, silicate dust processing and evolutionary stage versus age, comparing to similar low-mass star-forming regions, especially the young Coronet cluster, also known as CrA ( 1 Myr; Sicilia-Aguilar et al. 2008, from now on SA08, and references therein). The Coronet cluster shares with the $\eta$ Cha cluster a location, out of the galactic plane (De Zeeuw et al. 1999) and a population composed mainly of low-mass and very low-mass stars and BD, with only two intermediate-mass members (spectral types BA). Having very similar environment and population characteristics, the young CrA and the intermediate-aged $\eta$ Cha cluster seem to be "twins" at different stages of evolution. They are an ideal pair to study the effect of age on protoplanetary disks, separating the pure time evolution from the effects of the environment, initial conditions, and stellar mass. In Section 2 we briefly introduce the IRS and MIPS observations. In Section 3 we study the disk structure and dust composition of individuals in the global cluster context, examining the case of the B9 star $\eta$ Cha itself. Finally, our results are summarized in Section 4 ,

\section{Observations and Data Reduction}

We obtained $7.5-35 \mu \mathrm{m}$ low-resolution $(R=60-120)$ of the $\eta$ Cha cluster members (see Table 1) with the Infrared Spectrograph (IRS, Houck et al. 2004) on-board the Spitzer Space Telescope. A high accuracy optical peak-up (PCRS) was executed prior to the spectroscopic observations to position the target within the slit. Integration times were set such that the stellar photospheres could be detected with $\mathrm{S} / \mathrm{N} \sim 5$. All targets have been observed with a minimum of three observing cycles for redundancy.

Our spectra are based on the droopres products processed through the S15.3.0 version of the Spitzer data pipeline. Pixels flagged by the data pipeline as "bad" were replaced with a value interpolated from an 8 pixel perimeter surrounding the errant pixel. Partially based on the SMART software package (Higdon et al. 2004), our data were further processed using the spectral extraction tools developed for the "Formation and Evolution of Planetary Systems" (FEPS) Spitzer science legacy team (see also Bouwman et al. 2008). The spectra were extracted using a 6.0 pixel and 5.0 pixel fixed-width aperture in the spatial dimension for the observations with the first order of the short- $(7.5-14 \mu \mathrm{m})$ and the long-wavelength $(14-35 \mu \mathrm{m})$ modules, respectively. The background was subtracted using associated pairs of imaged spectra from the two nodded positions along the slit, also eliminating stray light contamination and anomalous dark currents. The low-level fringing at wavelengths $>20 \mu \mathrm{m}$ was removed using the irsfinge package (Lahuis \& Boogert 2003). To remove any effect of pointing offsets, we matched orders based on the point spread function of the IRS instrument, correcting for possible flux losses (see Swain et al. 2008 for further details).

The spectra were calibrated using a spectral response function derived from multiple IRS spectra of the calibration star $\eta^{1}$ Doradus and a MARCS stellar model provided by the Spitzer Science Center. The spectra of the calibration target were extracted in an identical way as our science targets. The relative errors between spectral points within one order are dominated by the 
noise on each individual point and not by the calibration. We estimate a relative flux calibration across an order of $\approx 1 \%$ and an absolute calibration error between orders/modules of $\approx 3 \%$, which is mainly due to uncertainties in the scaling of the MARCS model.

The $\eta$ Cha cluster was mapped using the Multiband Imaging Photometer (MIPS) on Spitzer. The observations were carried out on 2005 April 08 as part of the program number 100, using a medium scan map mode with half-array offsets. The mapped area of around $0.5^{\circ} \times 0.5^{\circ}$ covered most of the known $\eta$ Cha members. The MIPS photometry for the $\eta$ Cha members was presented by Gautier et al. (2008), but we found a systematic disagreement between their fluxes and our IRS observations that could not be explained with the typical uncertainties in these high signal-to-noise data. Therefore, we constructed the mosaics from the BCD data and repeated the photometry as standard aperture photometry. We analyzed the most recent calibrated data (BCD version S16.1.0). The mosaics at 24 and $70 \mu \mathrm{m}$ were created using the MOPEX software package (version 16.2.1). Taking into account the typical overlap per channel, the total exposure times were of $107 \mathrm{~s}$ and 54s at 24 and $70 \mu \mathrm{m}$, respectively. We used apertures of 13" and 30" for the 24 and $70 \mu \mathrm{m}$ photometry, respectively. The sky was measured in an annulus between 20"-32" for $24 \mu \mathrm{m}$ and 40"-60" for $70 \mu \mathrm{m}$, and we applied aperture corrections of 1.167 and 1.295 (for more details, see the MIPS Data Handbook 1). The measured MIPS magnitudes are listed in Table 3 . The uncertainties represent only the $1 \sigma$ statistical error without including further potential sources of errors that may sum up to $\sim 10 \%$ of the flux, depending on the location within the chip, flat fielding, and calibration (see Spitzer MIPS manual). The agreement between the IRS fluxes and our newly calculated MIPS magnitudes is very good, with typical errors within few percent.

In order to complete the spectral energy distributions (SEDs; Figures 1 and 2) and to compare the IR excess emission with the presence of gas accretion, we also include the data available for the cluster members in the literature (see Tables 1, 2, and 3). Optical magnitudes and spectral types are taken from Johnson et al. (1966), Mermilliod (1991), Mamajek et al. (1999), Lawson et al. (2001, 2002, 2004), and Lyo et al. (2003, 2004a, 2004b). Near-IR magnitudes from 2MASS (Cutri et al. 2003) are also available, as well as IRAC magnitudes (Megeath et al. 2005). Figure 3 shows the JHK and IRAC color-color diagrams for all the $\eta$ Cha cluster members.

Finally, for the purpose of modeling the underlying stellar atmosphere, a small grid of theoretical spectra was generated using the MARCS model atmosphere code (version 1998, Plez et al., 1992, and references therein). Detailed photospheric models, including IR opacities, are particularly important to study the excesses over the photospheric emission, which may be very small for the typical flattened disks around low-mass stars. The MARCS-code is aimed at modeling the atmospheres of cool stars, allowing for both plane-parallel and spherical geometries. Basic assumptions are local thermodynamic equilibrium (LTE), hydrostatic equilibrium, and conservation of energy for radiative and convective flux. The SEDs for the MARCS model atmospheres were calculated with the TURBospeCtRum code (Plez et al. 1992) using the same physical input parameters of

\footnotetext{
${ }^{1}$ http://ssc.spitzer.caltech.edu/mips/dh
} 
atomic and molecular equilibrium constants, solar abundances, and continuous opacity source, as described in Decin et al. (1998, 2000). For the line opacities, an infrared database including CO, $\mathrm{SiO}, \mathrm{H}_{2} \mathrm{O}, \mathrm{OH}, \mathrm{NH}, \mathrm{HF}, \mathrm{HCl}, \mathrm{CH}$, and $\mathrm{NO}$ was prepared (references and discussion can be found in Decin 2000). The grid of stellar spectra was generated for effective temperatures between 3200 and $4100 \mathrm{~K}$ (in steps of $300 \mathrm{~K}$ ) and gravity values $\left(\log \mathrm{g}\left[\mathrm{cm} / \mathrm{s}^{2}\right]\right.$ ) of 4.0, 4.5, 5.1, 5.6, and 6.10. A solar metallicity was assumed and the computations were performed for a plane-parallel geometry due

to the high gravity values. The synthetic spectra were derived for a resolution of $0.1 \AA$ and then degraded to the IRS resolution $(\mathrm{R} \sim 100)$. The MARCS models for the low-mass stars are displayed, together with the data, in Figures 1 and 2 ,

\section{Analysis and discussion}

\subsection{Disks around low-mass stars: Are the "transition" disks really in transition?}

Among the 15 low-mass members in the $\eta$ Cha cluster (spectral types K5.5-M5.5), we find 8 objects with IR excesses. Two of them (RECX-11, J0843) display the typical SEDs of classical T Tauri stars (CTTS) or Class II objects with flared disks, consistent with the Taurus median SED (including quartiles). Two more (J0841, J0844) are Class II objects with optically thick, but geometrically thin (flattened) disks (SED slope $\lambda \mathrm{F}_{\lambda} \sim \lambda^{-4 / 3}$; Hartmann 1998) and maybe even optically thin inner disks. Four objects (RECX-3, RECX-4, RECX-5, RECX-9) have inner opacity holes (no excess at wavelengths shorter than $6 \mu \mathrm{m}$ ), being empirically classified as "transition" objects (TO). The remaining 7 members have no significant IR excess, being Class III objects (see Figures 1, 2, 3).

Detecting inner holes in very low-mass stars with cold photospheres is more uncertain than in solar-type objects (spectral type K-early M), as the disk IR excesses tend to start at longer wavelengths and depend strongly on the size and angle of view of the disk inner rim (as it happens for solar-type stars with the $\mathrm{K}$ band excess). Therefore, we label as TO those objects with no significant excess at $\lambda<6 \mu \mathrm{m}$. This is an observational criterion, and it may not be sensitive to very small inner holes around the higher-mass stars. Some of the most flattened disks around late-M stars may have very small or negligible excesses around $6 \mu \mathrm{m}$ despite having optically thick disks without holes, as recent models have shown (Ercolano et al. 2009). Nevertheless, the contrast between solar-type and M-type stars is less dramatic if we assume more realistic temperatures for both types of objects, e.g. $\sim 4400 \mathrm{~K}$ for 1 Myr-old solar-type stars (a young, $\sim 1 \mathrm{M}_{\odot}$ star has an spectral type $\sim \mathrm{K} 5-\mathrm{K} 6$ ) and $\sim 3400 \mathrm{~K}$ for the M-type stars (e.g., Siess et al. 2000), instead of the values of $5000 \mathrm{~K}$ and $3000 \mathrm{~K}$ used by Ercolano et al. (2009). Flattened disks have historically been labeled as TO as well, given their differences with the typical, flared disks (e.g. Lada et al. 2006; Merín et al. 2008). Currie et al. (2009) defined these objects with flattened disks as "homologously depleted" transition disks, which should be considered as part of the TO family, given that their reduced IR excess involves substantial disk evolution with respect to flared Class II objects. This 
classification would include objects like J0841 in the TO family, and J0844 would be a border-line case. In addition, it is not known whether all disks evolve from Class II to Class III objects via the opening of an inner hole, and evolution may occur over a larger radial distance.

Two of the TO have small excesses (RECX-4 and RECX-3) that could also correspond to optically thin "debris" disks (Kenyon \& Bromley 2005). There are so far no statistical studies of the luminosity and evolution of debris disks around very low-mass stars. Some studies, based on millimeter data, suggest that debris disks are as common for M-type stars as they are for intermediate-mass stars (e.g., Lestrade et al. 2006), but they are based on a small number of objects, typically much older than the $\eta$ Cha cluster members. Distinguishing debris disks from TO for M-type objects with IR observations is not exent of problems. Unlike in more massive stars, the processes resulting in dust removing (e.g., Poynting-Robertson drag) require longer time to clean the disk ( $\sim 1 \mathrm{Myr})$, so objects with small excesses do not necessarily have second-generation dust (Currie et al. 2009). Therefore, within the limitations of small-number statistics, the estimated disk fraction is $\sim 50 \%$, and about half of the disks are TO, having probably cleaned or optically thin inner disks.

This disk fraction is similar to the values observed in younger clusters like the 4 Myr-old Tr 37 ( $\sim 50 \%$; Sicilia-Aguilar et al. 2006a 2 ), and higher than what we would expect for a $\sim 8$ Myr-old cluster (Haisch et al. 2001; Hernández et al. 2007). Nevertheless, most studies have concentrated on solar-type stars, and in any case, there is not much information about clusters older than 4 Myr. The fact that most of the low-mass stars in the $\eta$ Cha cluster have spectral types later than M0 could explain differences in disk mass, disk lifetimes, disk fractions, and disk structure/morphology if these parameters are related to the stellar mass. In addition, most of the clusters that have been analyzed to trace the evolution of the disk fraction are massive star-forming regions, containing several high- and intermediate-mass stars and hundreds of low-mass stars. The $\eta$ Cha cluster is very different, as it contains only 18 known members, most of them low-mass stars. If the environment and/or initial conditions have an influence in the formation of the disks and their subsequent evolution, we may expect differences between the disks characteristics and disk fraction in high-mass versus low-mass clusters.

TO represent usually only a small fraction $(\sim 5-10 \%)$ of the total number of disks (Hartmann et al. 2005; Sicilia-Aguilar et al. 2006a; Hernández et al. 2007). Nevertheless, our study of the young ( $\sim 1 \mathrm{Myr}$ ) Coronet cluster (SA08) revealed also a large fraction of TO $(\sim 50 \%$ of the total number of disks applying our observational criterion and including "homologously depleted" TO). The combined results of the $\eta$ Cha and Coronet clusters suggest that disks with opacity inner holes may be as (or nearly as) frequent as CTTS-like, optically thick disks for these late-type TTS at

\footnotetext{
${ }^{2}$ Note that this study is not complete at $24 \mu \mathrm{m}$, so objects with large holes similar to RECX-3 and RECX-4 are not included in the disk fraction for Tr 37. This can be generalized to many of the existing disk surveys, including those mentioned in Section 3.2 Complete surveys at $24 \mu \mathrm{m}$ would probably reveal larger disk fractions for these regions.
} 
any age. Recently, it has been suggested that our high TO fraction in the Coronet cluster was inaccurate (Ercolano et al. 2009), and most of the disks could be fitted by optically thick flattened SEDs without inner holes, leaving a TO fraction not different from observed around solar-type stars. This study provides valuable informtion that could be also applied to our results in the $\eta$ Cha cluster. While both the Coronet cluster and the $\eta$ Cha cluster are limited by low-number statistics given their small populations, we believe that TO fractions are higher than for solar-type stars, even if the SEDs of some objects may indeed be reproduced by models without holes. In particular, we strongly disagree with the model presented for CrA-205 by Ercolano et al. (2009), as it ignores the near-IR data on the basis of low $\mathrm{S} / \mathrm{N}$, and predicts a flux at $\sim 8 \mu \mathrm{m}$ that is more than $5 \sigma$ higher than both our IRS and IRAC observations. Their models for several objects (G-14, CrA-4111, and also CrA-205) require as well stellar luminosities and extinctions that differ from the observed values by more than the typical uncertainties, which suggests further model limitations. Therefore, we obtain a TO fraction of at least $\sim 30 \% \pm 10 \%$ for the Coronet cluster, higher if we include the homologously depleted "transition" objects and the objects with small excesses at $\lambda>20 \mu \mathrm{m}(\mathrm{G}-30, \mathrm{G}-94, \mathrm{G}-95$, and G-102).

A high fraction of TO seems to be present as well in the MBM12 cluster (Meeus et al. 2009). With a small population (like $\eta$ Cha and the Coronet cluster), an age of $2 \mathrm{Myr}$, and a very high disk frequency $(\sim 90 \%)$, about $40 \%$ of the disks in the MBM12 cluster resemble TO. The high number of flattened/transitional disks seems then ubiquitous for low-mass stars in small clusters of different ages, suggesting that their lifetimes are comparable to the lifetimes of the CTTS-like, optically thick disks. Therefore, at least some of the TO may not be in a short-lived stage between Class II and Class III objects, but may spend a large part of their lives as such structures.

The main reason to treat TO as something different from CTTS-like protoplanetary disks is their assumed short life. Since usually only $5-10 \%$ of the disks around solar-type stars are TO, their lifetimes are thought to be shorter than the lifetimes of "normal" protoplanetary disks. In such scenario, TO would be in a rapid, "transitional" stage between Class II and Class III objects, and the whole disk would disperse within $10^{5}-10^{6}$ yr after the innermost disk has been cleared. This could be achieved either by photoevaporation (Alexander et al. 2006a,b) and/or by planet formation (Quillen et al. 2004). More recently, it became evident that, although some TO harbor planets (e.g. TW Hya; Setiawan et al. 2008), others may have their inner part cleared since their formation due to the presence of close-in binaries (e.g. CoKu Tau/4; Ireland \& Kraus 2008). Although the structure of disks around M-type stars and even BD is sometimes considered as a smaller-scale version of solar-type TTS, disk masses around very low-mass objects are not well constrained, and the properties and structure of the innermost disk could be different if the disk masses are lower than in disks around solar-type stars (Hartmann et al. 2006). Therefore, the "transition" morphology may be due in some cases to other causes, instead of being an evolutionary process in an initially "normal" disk. In this case, the characterization of the properties of TO is needed to understand the disk physics.

The accretion rates can be used to test the planet formation and photoevaporation scenarios 
for TO. Considering the disk masses and accretion rates of the TO in Taurus, Najita et al. (2007) concluded that most of them are consistent with inner disk clearing by the formation of Jupitersized planets, being roughly one order of magnitude lower than the typical accretion rates for Class II objects in the region (Lubow \& D'Angelo 2006). The two accreting TO in $\eta$ Cha (RECX-5 and RECX-9) have very low accretion rates (see Table 1). Nevertheless, these accretion rates are not significantly different from those of the accreting Class II objects (J0843 and RECX-11), so in this case we cannot rule out nor confirm the planet formation scenario. On the other hand, the accretion rate of RECX-11 $\left(\dot{\mathrm{M}} \sim 410^{-11} \mathrm{M}_{\odot} / \mathrm{yr}\right)$ is well under the limit at which photoevaporation should become efficient to clean the innermost disk in short time $\left(\dot{\mathrm{M}} \sim 410^{-10} \mathrm{M}_{\odot} / \mathrm{yr}\right.$; Hollenbach et al. 2000). The fact that RECX-11 does not present an inner hole suggest that either the UV luminosities of young stars have been overestimated, or that the efficiency of photoevaporation is somehow reduced (for instance, because of gas shielding by the dust).

Regarding the presence of stellar companions to explain the TO, we do not have information about the Coronet cluster members, but several $\eta$ Cha members are known to be multiple systems (Table 1). In $\eta$ Cha, Bouwman et al. (2006) found that the presence of disks and nearby companions are anticorrelated, as the lifetimes of disks around binary systems are lower than for single stars. RECX-9 is probably the widest binary in the cluster, and the only with a (circumprimary) disk. Though the projected separation is only $20 \mathrm{AU}$, no motion has been detected in the system (Bouwman et al. 2006). A previous low-resolution spectroscopic study found a spectroscopic binary around RECX-7 with a period of 2.6 days (Moraux et al. 2007; Lyo et al. 2003). Nevertheless, the presence of spectroscopic binaries (which are the ones that could be responsible for the holes with sizes of few tenths of AU to a few AU detected at Spitzer wavelengths) around the rest of the $\eta$ Cha cluster members has not been explored yet.

\subsection{Evolution of the disks SEDs for low-mass objects}

The differences in disk morphology of the $\eta$ Cha cluster members compared to other TTS disks are reflected in the typical spectral energy distributions (SEDs), which are very different from what is observed for solar-type stars in other regions (Sicilia-Aguilar et al. 2006a; Figure 4 left). The median SED of the $\eta$ Cha disks around low-mass members (Figure 4 right) strongly differs from the typical SED for Taurus disks, falling below the SED of an optically thick, geometrically thin disk $\left(\lambda \mathrm{F}_{\lambda} \sim \lambda^{-4 / 3}\right.$; Hartmann 1998). This is indicative of a high degree of dust evolution/disk cleaning in the innermost disk. A similar median slope is also found in the young Coronet cluster (Figure 4 right; SA08). The median spectral type for objects with disks in both the $\eta$ Cha and the Coronet clusters is M3-M4.

In order to reveal the possible differences in disk evolution versus stellar mass and environment, we traced the median disk SED for M stars in several regions for which accurate spectral types, extinctions, and Spitzer data were available: the Coronet cluster (13 objects, median spectral type M4, 1 Myr; SA08), Taurus (23 objects, median spectral type M3, 1-2 Myr; Kenyon \& Hartmann 
1995; Briceño et al. 1997; Hartmann et al. 2005), IC 348 (128 objects, median spectral type M4, 2-3 Myr; Luhman et al. 2003; Lada et al. 2006), and 25 Ori (4 objects, median spectral type M3, 10 Myr; Briceño et al. 2007; Hernández et al. 2007). For each region, we considered all the objects with IR excesses and known spectral types (which are the majority of the disk members), including TO. The IRAC data on all these regions are complete (detecting diskless photospheres in all the mass range). The MIPS $24 \mu \mathrm{m}$ data are complete only for the $\eta$ Cha and the Coronet clusters. Therefore, the median points at $24 \mu \mathrm{m}$ for IC 348 and 25 Ori are upper limits, which may explain why the IC $34824 \mu \mathrm{m}$ value is much higher here than in the other regions.

The median SEDs were computed after correcting for the individual extinction of all objects in each region. For Taurus and 25 Ori, we used the average $A_{V}=1$ mag and $A_{V}=0.29 \mathrm{mag}$, respectively, for the objects with unknown extinction. Since the typical extinctions are low in both regions, individual variations are not significant at IR wavelengths. The median SEDs for low-mass objects (M0-M8) are displayed in Figure 4 (right). All the regions, except Taurus, show a median SED at $\lambda<6 \mu \mathrm{m}$ consistent with or lower than an optically thick, geometrically thin disk $\left(\lambda \mathrm{F}_{\lambda} \sim \lambda^{-4 / 3}\right)$. For 25 Ori, 3 of the disks are TO with no excess at $\lambda<8 \mu \mathrm{m}$. Solar-type objects in the same regions have different median disk SEDs (Lada et al. 2006), which is consistent with differences in disk structure and/or evolution depending on the spectral type.

This suggests that flattened (geometrically thin) and "transition" disks are more common among the low-mass objects than among solar-type stars in most regions, as previously suggested by Megeath et al. (2005), McCabe et al. (2006), and our observations of early M-type stars in the Cep OB2 region (Sicilia-Aguilar et al. 2007). One reason for this behavior could be that a given IR wavelength traces a smaller and closer-in zone in a mid- or late-M star than in a solar-type star, so any signs of inner disk evolution (grain growth, flattening, inner holes) will drastically reduce the IR excess (Kessler-Silacci et al. 2007). On the other hand, the fact that the median SEDs of $\eta$ Cha and the Coronet cluster are not significantly different at $\lambda<10 \mu \mathrm{m}$, and very flat at all wavelengths, suggests that the inner disk structure for the disks around low-mass objects differs from their solar-type counterparts at all ages. Differences in the disk structure (maybe because of the lower disk masses and the lack of dead zones; Hartmann et al. 2006) and/or initial conditions for the low-mass objects (for instance, in the angular momentum of the cores that form very lowmass stars; Dullemond et al. 2006) may be responsible for this effect. In addition, the environment (high-mass versus low-mass star forming regions) and the initial conditions in the star-forming cloud could affect both the formation and the evolution of the objects and their disks. This might explain why regions like Taurus (containing a relatively numerous population of low-mass stars in an undisturbed, quiescent environment, compared to OB associations) deviate from the flat-disk general trend seen in other clusters. 


\subsection{Dust mineralogy in the disk atmosphere: Grain processing and radial mixing}

The optically thin silicate emission seen at $\sim 10 \mu \mathrm{m}$ and $\sim 20 \mu \mathrm{m}$ in the IRS spectra traces the dust in the optically thin, warm $(\mathrm{T} \sim 150-450 \mathrm{~K})$ disk atmosphere (Calvet et al. 1992). Although this is only a very small part of the total dust contained in the disk, the presence of processed grains in the disk atmosphere reveals grain evolution (compared to ISM dust), grain transport in the disk, and the effect of turbulence. Among the $\eta$ Cha cluster members, only 5 show silicate emission over the continuum at $10 \mu \mathrm{m}$, and from those, only 4 display a clear silicate feature with good enough $\mathrm{S} / \mathrm{N}$ to allow a detailed analysis (RECX-5, RECX-9, RECX-11, and J0843). The silicate feature is weak in J0844, and absent in J0841. The spectrum of $\eta$ Cha does not show any features. There are no evident gas lines in the $\sim 13-14 \mu \mathrm{m}$ region (Carr \& Najita 2008) at more than a $3 \sigma$ level.

To study the grain properties in the disk atmospheres, we used the Two Layer Temperature Distribution (TLTD) spectral decomposition routines (Juhász et al. 2009, hereafter J09). The model reproduces the silicate emission using the sum of a multicomponent continuum and the optically thin emission of a set of 4 different dust species (amorphous silicates with olivine and pyroxene stoichiometry, forsterite, silica) with sizes $0.1,1.5$, and $6.0 \mu \mathrm{m}$, and enstatite grains with sizes 0.1 and $1.5 \mu \mathrm{m}$ (Dorschner et al. 1995; Servoin \& Piriou 1973; Jäger et al. 1998; Henning \& Mutschke 1997; Preibisch et al. 1993). We excluded from the fit the large enstatite grains $(6.0 \mu \mathrm{m})$, since there is no evidence for them in our data. Moreover, their contribution resembles the continuum at $10 \mu \mathrm{m}$, and can mask gas features in the 13-14 $\mu \mathrm{m}$ region. We also excluded from the fit carbon grains, which had been previously considered by J09, since their contribution is only an additional featureless continuum and does not affect the final silicate composition. Note that the emission from large $(6 \mu \mathrm{m})$ grains of most species differs little from the continuum emission (J09), so it is detectable only in objects with $\mathrm{S} / \mathrm{N}>100-200$. Therefore, the comparison of abundances and crystallinity fractions is valid only for the grain sizes detected in each spectrum, which depends on the $\mathrm{S} / \mathrm{N}$.

Instead of assuming one or two given temperatures for the silicate and continuum, the TLTD model considers that the silicate and the continuum originate in regions (disk inner rim, disk midplane, and optically thin disk atmosphere) that are characterized by a distribution of temperatures instead of a single one. These temperatures are parametrized as a power law. The mass absorption coefficients are derived from the material optical constants using the theory of Distribution of Hollow Spheres for the crystalline dust (Min et al. 2005), and the classical Mie theory for spherical particles for the amorphous dust. The flux then is calculated as:

$$
F_{\nu}=F_{\nu, \text { cont }}+\sum_{i=1}^{N} \sum_{j=1}^{M} D_{i, j} \kappa_{i, j} \int_{\mathrm{T}_{\mathrm{a}, \max }}^{\mathrm{T}_{\mathrm{a}, \min }} \frac{2 \pi}{d^{2}} B_{\nu}(T) T^{\frac{2-q a}{q a}} d T
$$

where the flux in the continuum is: 


$$
\begin{aligned}
F_{\nu, \mathrm{cont}}=D_{0} \frac{\pi R_{\star}^{2}}{d^{2}} B_{\nu}\left(T_{\star}\right) & +D 1 \int_{\mathrm{T}_{\mathrm{r}, \max }}^{\mathrm{T}_{\mathrm{r}, \min }} \frac{2 \pi}{d^{2}} B_{\nu}(T) T^{\frac{2-q r}{q r}} d T \\
& +D 2 \int_{\mathrm{T}_{\mathrm{m}, \max }}^{\mathrm{T}_{\mathrm{m}, \min }} \frac{2 \pi}{d^{2}} B_{\nu}(T) T^{\frac{2-q m}{q m}} d T
\end{aligned}
$$

Here, $R_{*}$ and $T_{*}$ are the radius and effective temperature of the star, and $T_{a}, T_{r}$, and $T_{m}$ are the disk atmosphere, rim, and disk midplane temperatures, which vary between a minimum and maximum value, and are power laws of the radius (with exponents qa, qr, and qm, respectively). The exponents of the temperatures (qa, qr, qm) and the coefficients of each contribution $\left(D_{0}, D_{1}\right.$, $D_{2}$, and $D_{i, j}$ ) are fitted to the data using a genetic optimization algorithm (see details in J09). The errors in the IRS spectra are taken into account by adding random Gaussian noise to the original spectrum at the noise level, and then repeating the fit 100 times. The final silicate composition is obtained as the average of the whole set, and the asymmetric errors are derived from the standard deviation in both the positive and negative direction, as was done for the Coronet cluster (SA08). The total crystalline fraction and the average grain sizes with their errors are estimated in the same way.

Tables 45, and 6 list the outcome of the fits in terms of mass abundances of the different species, temperature of the silicate-producing region, average grain sizes of the amorphous and crystalline silicates, and percentage of crystalline grains versus total in the optically thin disk atmosphere. We only fitted the objects with evidence of silicate emission. The applied spectral analysis method assumes that the dust composition does not vary in the disk with radial distance (wavelength). Thus, in order to handle possible differences in radial composition, we splitted the IRS spectra in two intervals $(7-17 \mu \mathrm{m}$ and $17-35 \mu \mathrm{m})$, which were fitted separately. We fitted J0843, J0844, RECX-5, RECX-9, and RECX-11 in the 7-17 $\mu \mathrm{m}$ region, and all of them except J0844 for the long-wavelength region. The fits are displayed in Figures [5 and 6, for the short and long wavelength ranges, respectively. The TLTD model and the material constants used are able to reproduce the observed features without any systematic deviation suggestive of other dust components (see residuals in Figures 5 and 6).

By fitting the 7-17 $\mu \mathrm{m}$ range, we find that the grain sizes in the optically thin disk atmospheres of the $\eta$ Cha cluster members are in all cases very different from what is seen in the ISM. The average grain size is $\sim 1-3 \mu \mathrm{m}$, and there is a significant fraction of crystalline silicates. In the $10 \mu \mathrm{m}$ region, the crystalline fractions observed range between $\sim 30 \%$ for J0843 to $\sim 7 \%$ for RECX-11. In general, the crystalline fraction is higher than observed around solar-type stars (Sicilia-Aguilar et al. 2007), and comparable to those observed in low-mass stars and BD (Apai et al. 2005; SA08). In lowmass objects, the $10 \mu \mathrm{m}$ feature samples a hotter, closer-in region than in solar-type stars. This may, at least in part, be the cause of these differences, if crystalline silicates are produced in the innermost disk. Large crystalline grains are rare (probably because they form large aggregates together with amorphous grains; Bouwman et al. 2008), and the emission from large amorphous 
grains $(>6-10 \mu \mathrm{m})$ resembles the continuum at $10 \mu \mathrm{m}$. Therefore, grain growth can also increase the measured crystalline fraction by "hiding" the amorphous dust into few-micron-sized ( $>6 \mu \mathrm{m})$ grains. Moreover, large grains can be removed from the disk atmosphere with time as settling increases (Sicilia-Aguilar et al. 2007), so the proportions between small crystalline grains and large amorphous grains can vary with age. Strong grain growth (lack of grains $<6 \mu \mathrm{m}$ ), resulting in the absence of the $10 \mu \mathrm{m}$ silicate feature in optically thick, accreting disks has been observed for early M stars in intermediate-aged regions (Sicilia-Aguilar et al. 2007). The differences in the processes producing crystalline silicates and in the regions where crystallinization occurs in solar-type and low-mass stars could also affect the crystalline fractions observed, as well as the properties of the crystals. In addition, if the disks of low- and very low-mass objects show structural differences (Hartmann et al. 2006), also the radial transport of processed grains in the disk could be affected.

The silicate features in the $17-35 \mu \mathrm{m}$ region is sensitive to colder dust and thus traces larger radial distances than the $10 \mu \mathrm{m}$ feature. Unless grain processing and growth occur at a similar rate at all radii and/or radial mixing is very efficient, we would expect to observe a radial variation of the grain composition. For the objects with good $\mathrm{S} / \mathrm{N}$ in the 17-35 $\mu \mathrm{m}$ region (J0843, RECX5, RECX-9, RECX-11; see Table 4), the differences in grain sizes between both regions are not significant, except for RECX-11, which shows larger amorphous grains in the $10 \mu \mathrm{m}$ region than at longer wavelengths. Nevertheless, the crystalline fractions are in general different, with a tendency to larger crystalline fractions at shorter wavelengths (closer-in radial distances). Longward of $17 \mu \mathrm{m}$ it is hard to estimate the total mass of amorphous silicate grains, since they lack sharp features in this range. Therefore, the variations in crystallinity with radius must be handled with care. For RECX-5 we observe differences in the crystalline silicate composition with radius: The enstatite to forsterite ratio decreases with wavelength (i.e., decreases with radial distance). This is the opposite effect we would expect from the dust coagulation model by Gail $(1998,2004)$, and has been observed in other regions (Bouwman et al. 2008; Meeus et al. 2009), which suggests that the conversion from forsterite to enstatite in chemical equilibrium does not take place in these disks.

Despite the limitations of small number statistics, the differences in silicate composition with the radial distance point to the importance of high temperatures in dust processing, and suggest a not very efficient radial mixing throughout the disk. We must nevertheless remember that old clusters have usually very small disk fractions. Our results for the $\eta$ Cha cluster are complete in the sense that they include all the known cluster members, which have been studied in detail. Nevertheless, they comprise a too small population to extrapolate these results to other regions with similar ages. Extending these observations to a larger number of objects and to regions with different ages would be needed to study the variations in grain processing and radial mixing with the evolutionary stage of the disk and the age of the system. 


\subsection{Dust evolution? The $\eta$ Cha cluster and its younger "twin", the Coronet cluster}

As we mentioned before, the low-mass $\eta$ Cha cluster strongly resembles the young ( $\sim 1 \mathrm{Myr})$ Coronet cluster. Comparing $\eta$ Cha to the Coronet cluster, we observe very similar mineralogy and crystalline fractions for the low-mass members that display silicate features (SA08), confirming that the dust composition derived from the silicate features cannot be used as a proxy for evolutionary stage nor age. This is probably due to the fact that the silicate feature traces only a minimal part of the dust content in the disk upper layers. It also suggests that the first grain processing (growth to few- $\mu$ m sizes and crystallization) occurs at a very early stage in the disk. The typical spectral types of the cluster members are similar in both cases, although the Coronet cluster harbors two objects near or at the Hydrogen burning limit.

Although the derived mineralogy is similar for the $\eta$ Cha and the Coronet cluster members, we observe other evolutive signatures when we compare their IRS spectra. About half of the disks in the Coronet cluster do not show $10 \mu \mathrm{m}$ features, despite having an excess in the $10 \mu \mathrm{m}$ region and being optically thick, protoplanetary disks (taking into account their IR excess over the photosphere). In the $\eta$ Cha cluster, 5 out of the 6 optically thick disks around the low-mass objects show silicate emission at $10 \mu \mathrm{m}$. The lack of silicate emission has been considered as a sign of evolution via grain growth, as large $(>6-10 \mu \mathrm{m})$ grains produce only continuum emission in the $10 \mu \mathrm{m}$ region. Nevertheless, a counterintuitive correlation between the size of the grains and the age (with older objects showing more pristine silicate features produced by small amorphous grains) has been noticed among solar-type stars in the Cep OB2 region (Sicilia-Aguilar et al. 2007). This anticorrelation between grain size and age seems related to the stronger settling and lower turbulence levels/accretion rates expected at older ages, which would not allow large grains to remain in the upper disk atmosphere (Sicilia-Aguilar et al. 2007). The correlation between grain size and accretion rate observed in Cep OB2 (Sicilia-Aguilar et al. 2007) and the large grains detected in FU Ori objects (Quanz et al. 2007) points in the same direction. The comparison between the $\eta$ Cha cluster and the young Coronet cluster reveals now the same effect around lowmass (M-type) objects. From these observations, we can conclude that large grains would form very early in the disk lifetime (as suggested by Dullemond \& Dominik 2005), and the grain population in the disk atmosphere probably depends on the effect of turbulence in the dust settling.

Accretion rates in the $\eta$ Cha cluster are low (Lawson et al. 2004; Table 2). Although there are no measurements of the accretion rates in the Coronet cluster, the $\mathrm{H} \alpha$ observations and line profiles suggest higher accretion rates than for the $\eta$ Cha members and thus, more turbulent disks (SA08). This is also the general trend we would expect from the studies of accretion rate evolution versus age (Muzerolle et al. 2000; Sicilia-Aguilar et al. 2006b), given the $\sim 7 \mathrm{Myr}$ age difference between both regions. Within the $\eta$ Cha cluster, we do not see any significant correlation between the grain size and the accretion rate (Lawson et al. 2004), although there are only 4 objects with known accretion rates, which are all very small (see Table 1). The way settling and turbulence affect the the vertical grain distribution, the composition of the disk atmosphere, and the appearance

of the silicate feature is not clear (Dullemond \& Dominik 2008). Therefore, this trend should be 
further explored and contrasted with other stellar and environmental properties. Other parameters affecting the disk structure, like differences in stellar mass/spectral type, or differences in the disk mass, evolution, and environment, could be partially responsible for this effect.

Taking into account the IR photometry of the $\eta$ Cha members, it had been suggested that these disks would have suffered strong grain growth (Haisch et al. 2005; Megeath et al. 2005). While this is probably true, and may be responsible for the flattened SEDs and TO observed for the $\eta$ Cha members (see Section 3.2), it cannot be confirmed using the silicate emission at IR wavelengths. The large grains are most likely larger than a few $\mu \mathrm{m}$, and have probably settled to the disk midplane, so they are not present in the disk atmosphere, where the optically thin emission arises. In addition, IR observations are not sensitive to the bulk of the dust content in the disk, including the potential very large grains (more than a few $\mu \mathrm{m}$ in size, maybe even pebble-sized) that are likely to have formed in the $8 \mathrm{Myr}$ life of the disks around $\eta$ Cha members. Observations at millimeter and sub-millimeter wavelengths are needed to trace the presence and mass distribution of larger grains in these disks.

\section{5. $\quad \eta$ Cha: A young debris-disk rather than a classical Be star}

The B9 star $\eta$ Cha is the brightest member of the cluster that bears its name. Its fast rotation, together with the presence of a flattened, featureless disk, suggested that it could be a classical Be star (see the review by Porter \& Rivinius 2003). Traditionally, classical Be stars had been considered as an older stage in the evolution of very fast rotating B and early A stars. These stars would lose material at their equator, producing a gaseous hot disk that is bright in the IR via free-free emission (Gehrz et al. 1974). Initial surveys of young clusters suggested that there were no classical Be stars in regions aged $<10$ Myr (Capilla et al. 2000). Nevertheless, more recent studies revealed several classical Be stars in young regions (Bhavya et al. 2008). Even the $\sim 4$ Myr old cluster Tr 37 possesses a classical Be star, KUN-314s (Sicilia-Aguilar et al. 2007). Aged $\sim 8 \mathrm{Myr}, \eta$ Cha would be among the youngest Be stars known. Nevertheless, it could as well be a young debris disk, given that the excess emission over the stellar photosphere is comparable to what is seen in young debris disks a few Myr after planet formation (Kenyon \& Bromley 2005). In addition, there are known debris disks with mid-IR SED slopes similar to $\eta$ Cha (Sicilia-Aguilar et al. 2007).

To test both possibilities, we have compared the optical spectrum of $\eta$ Cha taken with UVES (from the ESO data archive) to the HIRES spectra of two young B stars in Tr 37 (kindly provided by L. Hillenbrand), the classical Be star KUN-314s and the B7 star MVA-437 (Contreras et al. 2002; Sicilia-Aguilar et al. 2005, 2007). $\eta$ Cha was observed with UVES under the ESO program ID 66.D-0284(A) in 2001-02-18. All the data were obtained with the DICHROIC\#2 mode, which has two wavelength settings, BLUE (305-385 nm) and RED (575-945 nm, split up in two wavelength ranges REDL and REDU). The 8 spectra (4 taken with the BLUE setting, and 4 taken with the RED setting) were reduced using the standard UVES pipeline. Integration times were short, 5-15 s 
for the red part, and $10 \mathrm{~s}$ for the blue, as $\eta$ Cha is extremely bright for UVES mounted on a UT. The final spectrum is the sum of these spectra. The HIRES spectra of KUN-314s and MVA-437 were taken in 2001, before the HIRES upgrade, using the rg610 filter, which provides a wavelength coverage between 6350 and $8750 \AA$ with a resolution $\sim 34,000$. The spectra were reduced using the MAKEE Keck Observatory HIRES data reduction software, developed by T. Barlow.

The optical spectra were scaled to match their continuum levels and compared to determine the presence of the emission lines typical of classical Be stars (Figure 7). The comparison reveals that $\eta$ Cha does not show any of the emission lines typical of classical Be stars like KUN-314s. In particular, it lacks the $\mathrm{H}$ and $\mathrm{He}$ emission lines with the double-peaked rotation profiles characteristic of classical Be stars. On the contrary, the spectrum of $\eta$ Cha is very similar to that of the B7 star MVA-437, except for the accretion-related $\mathrm{H} \alpha$ line, for which MVA-437 shows narrow emission but the non-accreting $\eta$ Cha displays only rotationally broadened absorption. Some differences in the absorption lines (line broadening, lack of He absorption lines in $\eta$ Cha) are due to differences in rotation and small differences in the spectral type, since $\eta$ Cha is a faster rotator than MVA-437 and a B9 star, instead of a B7 star like MVA-437.

Another reason that suggested that $\eta$ Cha could be a classical Be star was the slope of its SED, similar to the expected slope for free-free emission found in classical Be stars (Gehrz et al. 1974). KUN-314s is a late-B star (the uncertain spectral type is due to the double-peaked emission lines masking most of the A and B spectral type features), having thus a spectral type similar to $\eta$ Cha. Therefore, we would expect a very similar emission from a classical Be "decretion" disk if both objects have the same nature, with some variations depending on whether the decretion disk is optically thin or optically thick (Gehrz et al. 1974). For comparison, the SED of KUN-314s was scaled to match the distance and luminosity of $\eta$ Cha (Figure 8). The wavelength at which the IR excess starts is different in $\eta$ Cha and in KUN-314s. In fact, the entire $\eta$ Cha SED is more similar to the debris disk around the B7 star MVA-468. Both $\eta$ Cha and MVA-468 lack silicate and gas features, and have a slope similar to that expected from free-free emission, although the $70 \mu \mathrm{m}$ flux in $\eta$ Cha suggests a deviation from the free-free emission slope at long wavelengths. Debris and "transitional" disks with such slopes have been found not only around B-type stars, but also around F-type objects (e.g., MVA-447 in Tr 37; Sicilia-Aguilar et al. 2007). Although some of the debris and/or "transition" disks around intermediate-mass stars show silicate features in the 10-30 $\mu \mathrm{m}$ region, most of them display flat, featureless spectra (Sicilia-Aguilar et al. 2007), probably because of the lack of small $(<10 \mu \mathrm{m})$ dust grains. Due to its high luminosity, the silicate feature in a B9 star is produced by small grains located at up to $\sim 70-100$ AU distance, so the total absence of silicate emission in the IRS spectrum points to the lack of small grains in most or even in the whole disk. Then, B stars with featureless mid-IR spectra contain probably second-generation, collisional, reprocessed debris disks made of larger grains (Kenyon \& Bromley 2005).

Therefore, we conclude that there is no evidence of $\eta$ Cha being a classical Be star. Despite its fast rotation, it is not significantly different from a normal, young B star surrounded by a disk with a large inner hole. The disk could be either the leftover from an evolved protoplanetary 
disk that has been evacuated in its innermost regions by a non-detected companion, grain growth, photoevaporation, and/or planet formation ("transition" disk), or more likely, due to the lack of small dust grains, a debris disk made by reprocessed dust from collisions a few Myr after planet formation.

\section{Conclusions}

The IRS spectra of the $\eta$ Cha cluster members presented here cover probably the largest and best studied sample of disks in a $\sim 8 \mathrm{Myr}$ old cluster, and are particularly interesting to understand disk evolution, given that the stellar properties of the $\eta$ Cha members (spectral types, ages, accretion, and in most cases, binarity) are well known. Moreover, the $\eta$ Cha cluster is a key to understand disk evolution, as it still contains a large population of disks for its old age.

The IRS data confirms a relatively high disk fraction for a $\sim 8$ Myr-old cluster, $~ 50 \%$, among the low-mass stars (spectral types late K-M). Half of the disks are TO, which is a higher fraction than observed for solar-type stars in other regions (e.g., Taurus, $\operatorname{Tr} 37$, Orion), even if we consider the effects of small number statistics and the differences in luminosity between M-type and solartype stars. The fraction of TO is comparable to the TO fraction in the young ( $\sim 1 \mathrm{Myr})$, low-mass star Coronet cluster and in MBM12 ( $\sim 2 \mathrm{Myr})$. The large number of TO (including flattened, "homologously depleted" TO) in clusters with very different ages suggest that at least some TO are long-lived, and therefore, not in an intermediate, rapid "transitional" stage between Class II and Class III objects.

Disks around low-mass stars have lower masses than the disks around solar-type TTS, so they might also have different structure (Hartmann et al. 2006) and/or evolve along a different path than their solar-type companions. Long-lived "transition" objects do not fit within the classical picture of rapid disk dissipation, suggesting that they could be part of the "normal" type of disk around very low-mass objects. The presence of long-lived inner holes and depleted inner disks could be related to planetesimal or even planet formation, non-resolved binary companions, or be the result of the initial conditions (disk mass and structure) and formation environment for these low-mass objects. The TO in $\eta$ Cha have zero or very low accretion rates, which could be compatible with the planet formation hypothesis. In addition, the fact that one of the Class II disks has a very low accretion rate (RECX-11) and no inner hole suggests that photoevaporation, expected to remove disks in timescales $\sim 10^{5}$ years, may not be as efficient to open inner holes as previously thought. Further observations, especially those focused on the detection of close-in companions, are now required to study the origin and properties of accreting and non-accreting TO.

The IRS spectra of the low-mass cluster members also reveal that the dust grains in the optically thin disk atmospheres have suffered substantial grain processing, being very different from ISM grains. They are typically large (1-3 $\mu \mathrm{m})$ and contain different proportions of crystalline silicates ( 10-30\%) and a large variety of grain compositions. The differences between the 7-17 $\mu \mathrm{m}$ 
and the 17-35 $\mu \mathrm{m}$ features show differences in grain composition and solid state with the radial distance, suggesting inefficient radial mixing, and the importance of high temperatures in dust processing.

The diversity of cases, and the fact that the grains are not different from what is found in objects with similar spectral types in younger clusters (e.g. the Coronet cluster), suggest that the grain size/crystalline fraction observed cannot be used as a proxy for age nor evolutionary state. Few- $\mu \mathrm{m}$ grains and crystalline silicates must then form very early in the disk life. The grain population in the disk atmosphere is probably more affected by the turbulence levels in the disk than by the global grain growth. Therefore, objects with SEDs lacking silicate features, having presumably disk atmospheres dominated by large grains, appear to be more common in younger, more turbulent disks. IR observations are not sensitive to grains larger than $\sim 10 \mu \mathrm{m}$, whose mass fractions and sizes might be better tracers of the evolutionary state.

The combination of IRS, MIPS, and UVES data on the B9 star $\eta$ Cha that gives the name to the cluster reveals that it is surrounded by a debris disk or a very evolved protoplanetary disk with a large inner hole and no small dust grains, rather than being a young, classical Be star as it had been suggested. The luminosity of this disk is consistent with the predictions for a second-generation debris disk a few Myr after planet formation (Kenyon \& Bromley 2005).

Finally, we make available the corrected MIPS photometry for the $\eta$ Cha cluster members, since the data given in the literature was probably affected by errors in the data reduction process and/or in the photometry. Our revised MIPS photometry is fully consistent with the fluxes in the IRS data.

We want to thank L. Hillenbrand for providing the HIRES spectra of the intermediate-mass members of $\operatorname{Tr} 37, \mathrm{KUN}-314 \mathrm{~s}$ and MVA-437. We also thank the anonymous referee for his/her review, interesting suggestions and useful comments, which have greatly contributed to the clarity of this paper. A.S-A. acknowledges support from the Deutsche Forschungsgemeinschaft, DFG, grant number SI 1486/1-1. This work is based on observations made with the Spitzer Space Telescope, which is operated by the Jet Propulsion Laboratory, California Institute of Technology under a contract with NASA. It also makes use of data products from the Two Micron All Sky Survey, which is a joint project of the University of Massachusetts and the Infrared Processing and Analysis Center/California Institute of Technology, funded by the National Aeronautics and Space Administration and the National Science Foundation.

\section{REFERENCES}

Alexander, R., Clarke, C., Pringle, J, 2006a MNRAS, 369, 216

Alexander, R., Clarke, C., Pringle, J, 2006b MNRAS, 369, 229 
Apai, D.; Pascucci, I.; Bouwman, J.; Natta, A.; Henning, Th.; Dullemond, C. P., 2005, Sci, 310, 834

Bhavya, B., Blesson, M., Subramaniam, A., 2008, BASI in press

Bouwman, J.; Lawson, W. A.; Dominik, C.; Feigelson, E. D.; Henning, Th.; Tielens, A. G. G. M.; Waters, L. B. F. M., 2006, ApJ, 653, 57

Bouwman, J.; Henning, Th.; Hillenbrand, L. A.; Meyer, M. R.; Pascucci, I.; Carpenter, J.; Hines, D.; Kim, J. S.; Silverstone, M. D.; Hollenbach, D.; Wolf, S.

Brandeker, A., Jayawardhana, R., Khavari, P., Haisch, K., Mardones, D., 2006, ApJ 652, 1572

Briceño, C., Hartmann, L., Stauffer, J., Martín, E., 1998, AJ, 115, 2074

Briceño, C., Hartmann, L., et al., 2007, ApJ, 661, 1119

Calvet, N., et al. 1992, RMxAA 24, 27

Capilla, G.; Fabregat, J.; Baines, D.,2000, ASPC, 214, 63

Carr, John S.; Najita, Joan R., 2008, Science, 319, 1504

Contreras, M.E., Sicilia-Aguilar, A., Muzerolle, J., Calvet, N., Berlind, P., Hartmann, L. 2002, AJ, 124,1585

Currie, T., Lada, Ch., Plavchan, P., Robitaille, Th., Irwin, J., Kenyon, S., 2009, ApJ in press

Cutri R.M., Skrutskie, M.F., van Dyk, S. \& 25 more coauthors, 2003yCat.2246, 0C, VizieR On-line Data Catalog: II/246

Decin, L.; Van Winckel, H.; Waelkens, C.; Bakker, E. J., 1998, A\&A 332, 928

Decin, L.; Waelkens, C.; Eriksson, K.; Gustafsson, B.; Plez, B.; Sauval, A. J.; Van Assche, W.; Vandenbussche, B., 2000, A\&A 364, 137

Decin, L., PhD Thesis, , Catholique University of Leuven, 2000

De Zeeuw, P., et al. 1999, AJ, 117, 354

Dorschner, J, Begemann, B., Henning, T., Jäger, C., \& Mutschke, H., 1995, A\&A, 300, 503

Dullemond, C., \& Dominik, C., 2005, AA, 434, 975

Dullemond, C., Apai, D., Walch, S., 2006, ApJ in press

Dullemond, C., \& Dominik, C., 2008, A\&A, 487, 205

Ercolano, B., Clarke, C., Robitaille, T., 2009, MNRAS in press 
Gail, H.-P.,1998, A\&A 332, 1099

Gail, H.-P., 2004, A\&A 413, 571

Gautier, Thomas. N., III; Rebull, L. M.; Stapelfeldt, K. R.; Mainzer, A., 2008, ApJ 683, 813

Gehrz, R., Hackwell, J., Jones, T., 1974, ApJ, 191, 675

Haisch, K., Lada, E., \& Lada, C., 2001, ApJ, 553, 153

Haisch, K.E., Jayawardhana, R., \& Alves, J., 2005, ApJ 627, L57

Hartmann, L.: Accretion Processes in Star Formation, Cambridge University Press, 1998.

Hartmann, L., Megeath, S.T., Allen, L., Luhman, K., Calvet, N., D’Alessio, P., Franco-Hernández, R., Fazio, G., 2005, ApJ, 629, 881

Hartmann, L., D’Alessio, P., Calvet, N., \& Muzerolle, J., 2006, ApJ, 648, 484

Henning, Th.; Launhardt, R.; Steinacker, J.; Thamm, E., 1994, A\&A, 338, 223

Henning, Th.\& Mutschke, H., 1997, A\&A, 327, 743

Hernández, J., Hartmann, L., Megeath, S.T.,et al., 2007, ApJ, 662, 1067

Higdon, S., et al., 2004, PASP, 116, 975

Hollenbach, D., Yorke, H., Johnstone, D., 2000, Protostars and Planets IV, Univ. Arizona Press, p. 401

Houck, J., et al. 2004, ApJS, 154, 18

Jäger, C., Molster, F., Dorschner, J., et al., 1998, A\&A 339, 904

Johnson H.L., Mitchell R.I., Iriarte B., and Wisniewski W.Z., 1966, CoLPL,4,99

Juhász, A.; Henning, Th.; Bouwman, J.; Dullemond, C.; Pascucci, I.; Apai, D., 2009, ApJ in press (J09)

Ireland, M., Kraus, A., 2008, ApJ, 678, 59

Kenyon, S.J. \& Hartmann, L., 1995, ApJS , 101, 117

Kenyon, S.J., \& Bromley, B.C., 2005, AJ, 130, 1

Kessler-Silacci, J., et al., 2007, ApJ,659,680

Köhler, R., \& Petr-Gotzens, M., 2002, AJ 124, 2899

Lada, C., Muench, A., et al., 2006, AJ, 131, 1547 
Lahuis, F., Boogert, A., 2003cdsf.conf..335L

Lawson, Warrick A.; Crause, Lisa A.; Mamajek, Eric E.; Feigelson, Eric D., 2001, MNRAS, 321, 57

Lawson, Warrick A.; Crause, Lisa A.; Mamajek, Eric E.; Feigelson, Eric D., 2002, MNRAS, 329, 29

Lawson, W., Lyo, A., Muzerolle, J., 2004, MNRAS, 329, 29

Lestrade, J.F., Wyatt, M., Bertoldi, F., Dent, W., Menten, K., 2006, A\&A 460, 733

Luhman, K. L.; Stauffer, John R.; Muench, A. A.; Rieke, G. H.; Lada, E. A.; Bouvier, J.; Lada, C. J., 2003, ApJ, 593, 287

Luhman, K. L.; Steeghs, D., 2004, ApJ, 609, 917

Luhman, K.,2004, ApJ, 616, 1033

Luhman, K., Lada, C., Hartmann, L., et al., 2005, ApJ, 631, 69

Lyo, A.-R.; Lawson, W. A.; Mamajek, E. E.; Feigelson, E.; Sung, E.-C.; Crause, L. A., 2003, MNRAS, 338, 616

Lyo, A.-R.; Lawson, W. A.; Feigelson, E.; Crause, L. A., 2004a, MNRAS, 347, 246

Lyo, A.-R.; Song, I., Lawson, W.; Bessell, M., Zuckerman, B., 2004b, MNRAS, 368, 1451

Mamajek, E., Lawson, W., Feigelson, E., 1999, ApJ, 516, 77

Mamajek, E., Lawson, W., Feigelson, E., 2000, ApJ, 544, 356

McCabe, C., Ghez, A., Prato, L, Duchene, G., Fisher, R., Telesco, C., 2006, ApJ 636, 932

Meeus, G.; Juhasz, A.; Henning, Th.; Bouwman, J.; Chen, C.; Lawson, W.; Apai, D.; Pascucci, I.; Sicilia-Aguilar, A., 2009, A\&A in press

Megeath, S. T.; Hartmann, L.; Luhman, K. L.; Fazio, G. G., 2005, ApJ, 634, 113

Merín, B., et al. 2008, ApJS, 177, 551

Mermilliod J.C., 1991,Homogeneous Means in the UBV System, Institut d'Astronomie, Universite de Lausanne

Meyer, M. R., Calvet, N., \& Hillenbrand, L. A. 1997, AJ, 114, 288

Min, M.; Hovenier, J. W.; de Koter, A., 2005, A\&A 432, 909

Moraux, E.; Lawson, W. A.; Clarke, C., 2007, A\&A 473, 163 
Muzerolle, J., Calvet, N., Briceño, C., Hartmann, L. \& Hillenbrand, L., 2000, ApJ, 535, L47

Najita, J., Strom, S., Muzerolle, J., 2007, MNRAS, 378, 369

Plez, B., 1992, A\&AS, 94, 527

Porter, J., \& Rivinius, T., 2003, PASP, 115, 1153

Preibisch, T., Ossenkopf, V., Yorke, H., \& Henning, T., 1993, A\&A, 279, 577

Quanz, S., Henning, Th., Bouwman, J., Van Boekel, R., Juhasz, A., Linz, H., Pontoppidan, K., Lahuis, F., 2007, ApJ, 668, 359

Quillen, A., Blackman, E., Frank, A., Varniere, P., 2004, ApJ, 612, L137

Servoin, J., \& Piriou, B., 1973, Phys. Stat. Sol., 55, 677

Setiawan, J.; Henning, Th.; Launhardt, R.; Müller, A.; Weise, P.; Kürster, M., 2008, Nature, 451, 38

Sicilia-Aguilar, A., Hartmann, L., Calvet, N., Megeath, S.T., Muzerolle, J., Allen, L., D'Alessio, P., Merín, B., Stauffer, J., Young, E., Lada, C., 2006a,ApJ 638, 897

Sicilia-Aguilar, A., Hartmann, L., Fürész, G., Henning, Th., Dullemond, C., Brandner, W., 2006b, AJ 132, 2135

Sicilia-Aguilar, A., Hartmann, L., Watson, D., Bohac, C., Henning, Th., et al., 2007, ApJ 659, 1637

Sicilia-Aguilar, A.; Henning, Th.; Juhsz, A.; Bouwman, J.; Garmire, G.; Garmire, A., 2008, ApJ, 687,1145

Siess, L., Dufour, E. \& Forestini, M. 2000 A\&A , 358, 593

Swain, M., Bouwman, J., Akeson, R., Lawler, S., Beichman, C., 2008, ApJ 674, 482 


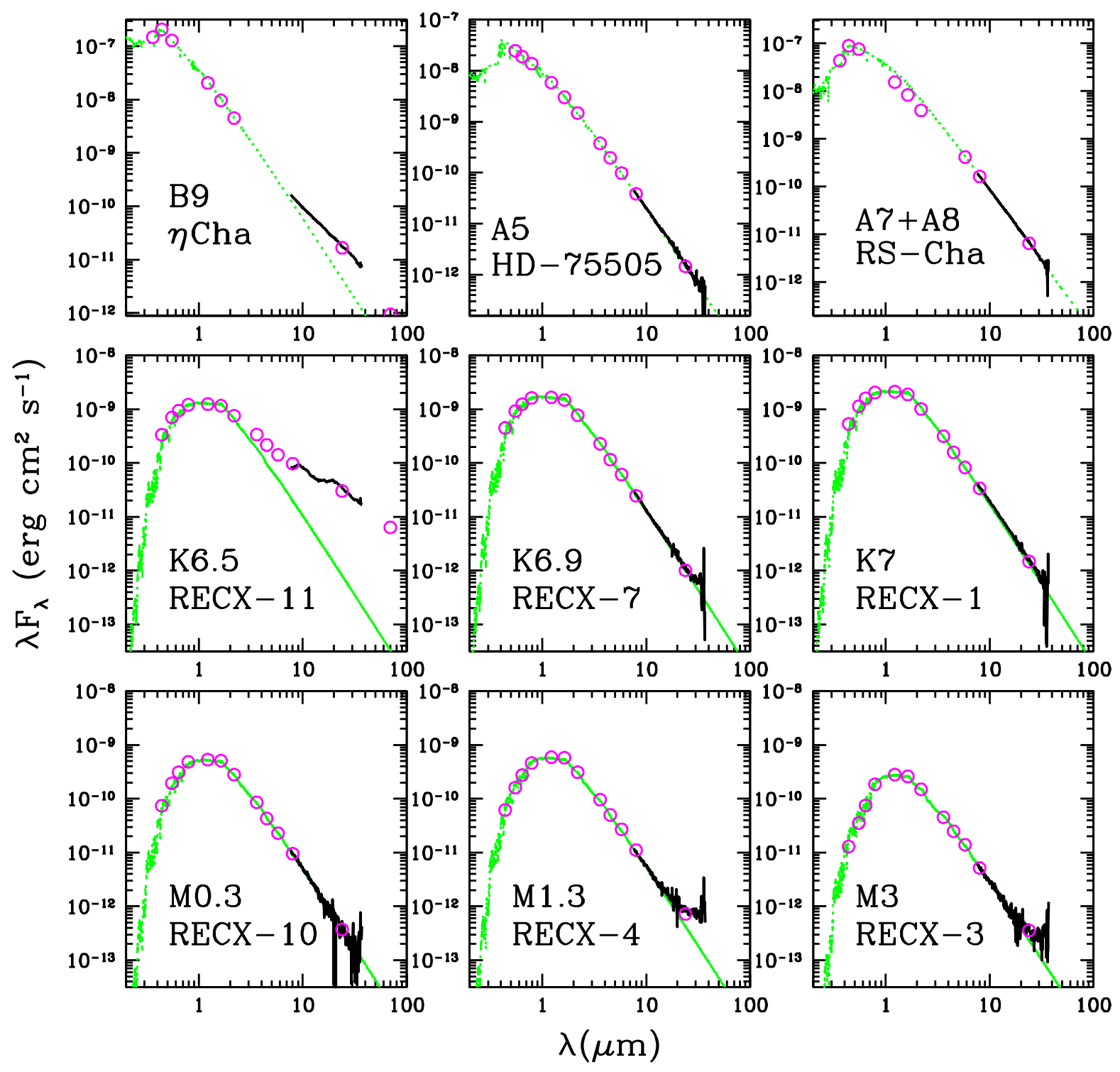

Fig. 1.- SEDs of the $\eta$ Cha members, including optical and IR data (open circles), IRS spectra (thick black line) and stellar models (dotted green line, including MARCS models for the low-mass stars). (see Tables 2 and 3). RS Cha is an eclipsing binary; the 2MASS data were obtained during an eclipse and accordingly fall under the photospheric model which fits the combined SED of both stars 


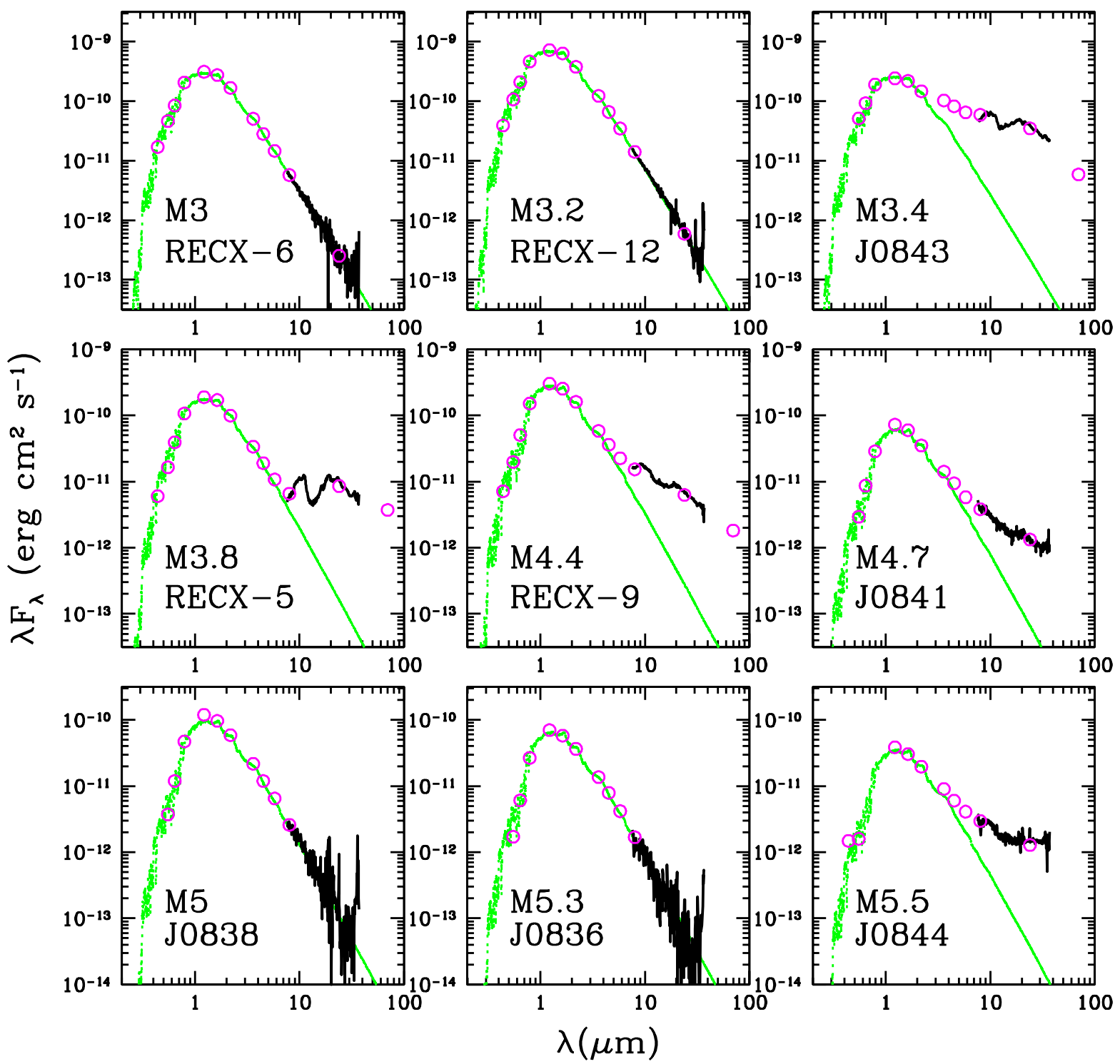

Fig. 2.- SEDs of the $\eta$ Cha members, including optical and IR data (open circles), IRS spectra (thick black line) and stellar models (dotted green line, including MARCs models for the low-mass stars). (see Tables 2 and 3 ). 

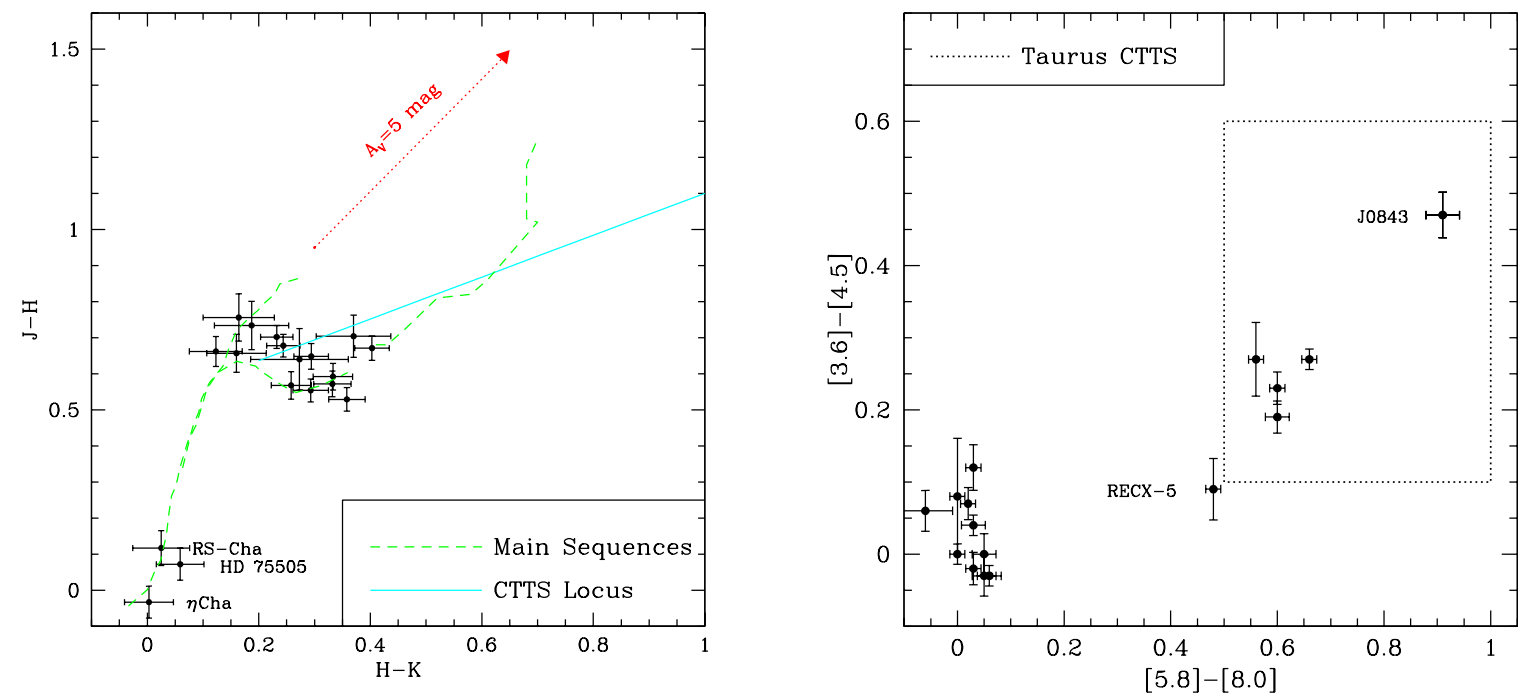

Fig. 3.- 2MASS and IRAC color-color diagrams for the $\eta$ Cha cluster. The JHK diagram shows the main sequences for dwarfs, giants (Bessell \& Brett 1989), and brown dwarfs (Kirkpatrick et al. 1996), and the locus of the classical T Tauri stars (CTTS locus; Meyer et al. 1997). RECX-5 shows typical colors of a TO with an excess starting after $\sim 5.8 \mu \mathrm{m}$, and J0843 presents a strong excess typical of a very flared disk. The four other object with colors consistent with Taurus CTTS are RECX-9, RECX-11, J0841, and J0844, but since their disks are flatter/transitional, the objects are less red than the typical Taurus CTTS. 

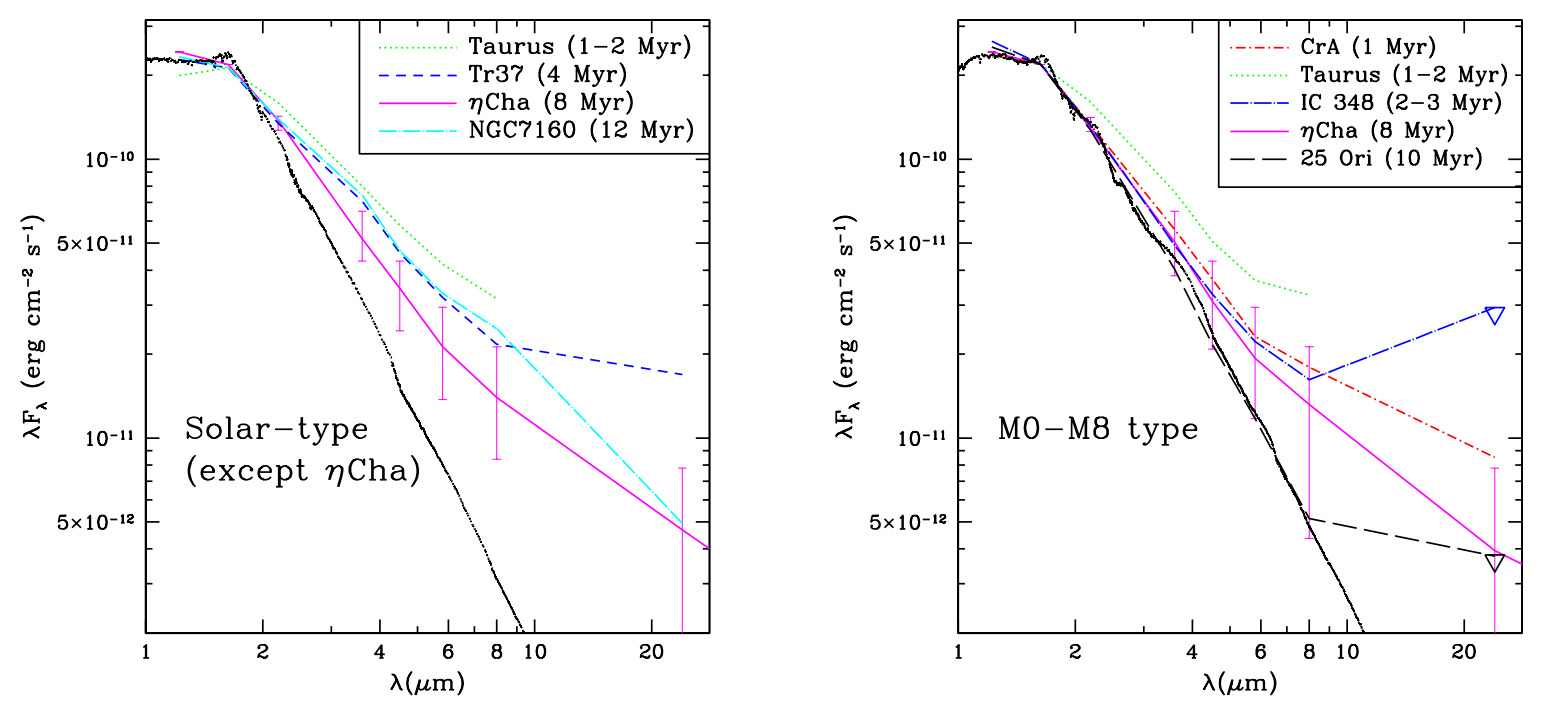

Fig. 4.- Median SED for $\eta$ Cha low-mass (M-type) members, compared to the median SEDs of different regions for solar-type (K-early M, left) and M-type objects (right) with different ages: $\mathrm{Tr}$ 37 and NGC 7160 (4 and 12 Myr, respectively; Sicilia-Aguilar et al. 2006); CrA (1 Myr; SA08); Taurus (1-2 Myr, Hartmann et al. 2005); IC 348 (2-3 Myr; Luhman et al. 2003; Lada et al. 2006); 25 Ori (10 Myr; Briceño et al. 2007; Hernández et al. 2007). All the colors have been dereddened using the corresponding extinctions, and the fluxes have been scaled to match the distance and luminosity of the $\eta$ Cha cluster. The median SEDs are compared to the photospheric emission of a K7 (left) and an M4 star (right, also from the MARCS models; dotted line). All the datasets are complete at IRAC wavelengths, MIPS $24 \mu \mathrm{m}$ is only complete for the $\eta$ Cha cluster and the Coronet cluster (see text); therefore it is marked with an inverted triangle as an upper limit in IC 348 and 25 Ori. The quartiles are displayed for the $\eta$ Cha cluster, being similar for the other regions. 


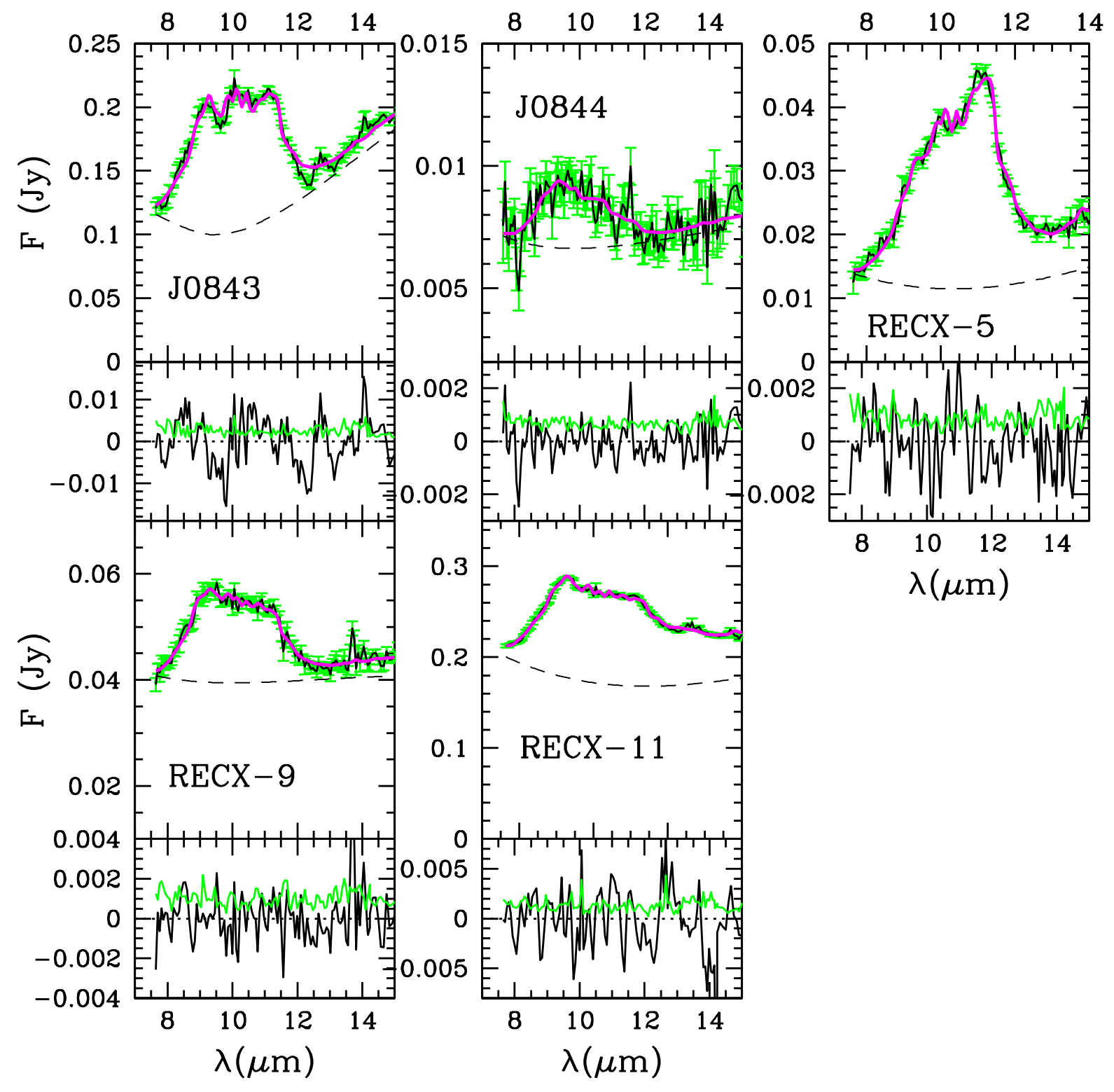

Fig. 5.- Silicate fitting of the $10 \mu \mathrm{m}$ feature of the stars in $\eta$ Cha, using the TLTD method. The original spectrum is represented as a black solid line, with the errors in green. The fit to the continuum is the dashed line, and the model fit is the magenta thick solid line. The residuals (black), together with the errors (green), are displayed under each source, and they do not reveal any systematic deviations. Note that the fit for J0844 only indicates the presence of silicate emission, but cannot be used to obtain details about mineralogy due to the low $\mathrm{S} / \mathrm{N}$ of the spectrum. 


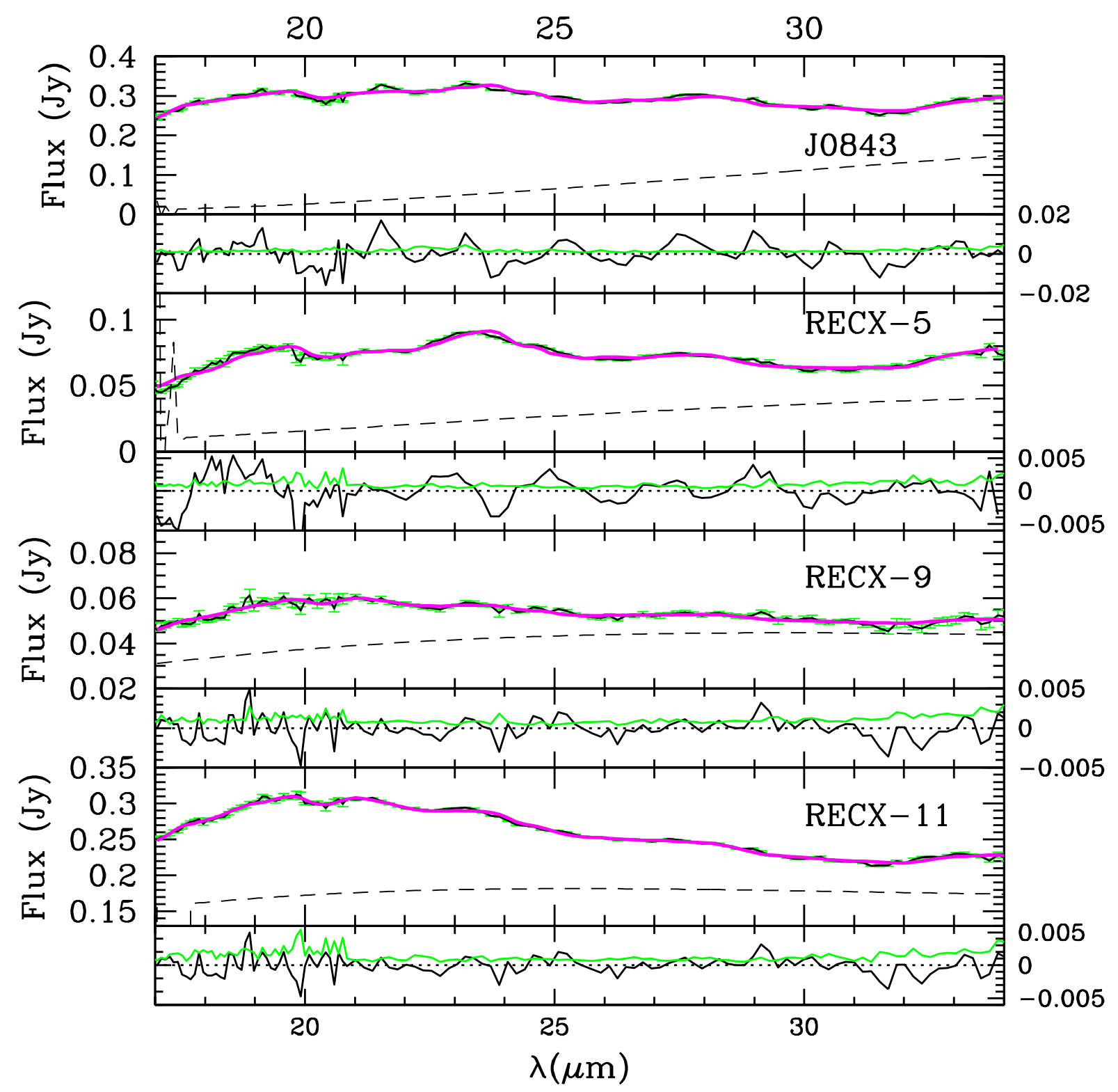

Fig. 6.- Silicate fitting at 17-35 $\mu \mathrm{m}$ for the stars in $\eta$ Cha, using the TLTD method. The original spectrum is represented as a black solid line, with the errors in green. The fit to the continuum is the dashed line, and the model fit is the magenta thick solid line. The residuals (black), together with the errors (green), are shown in the panel below each object. 

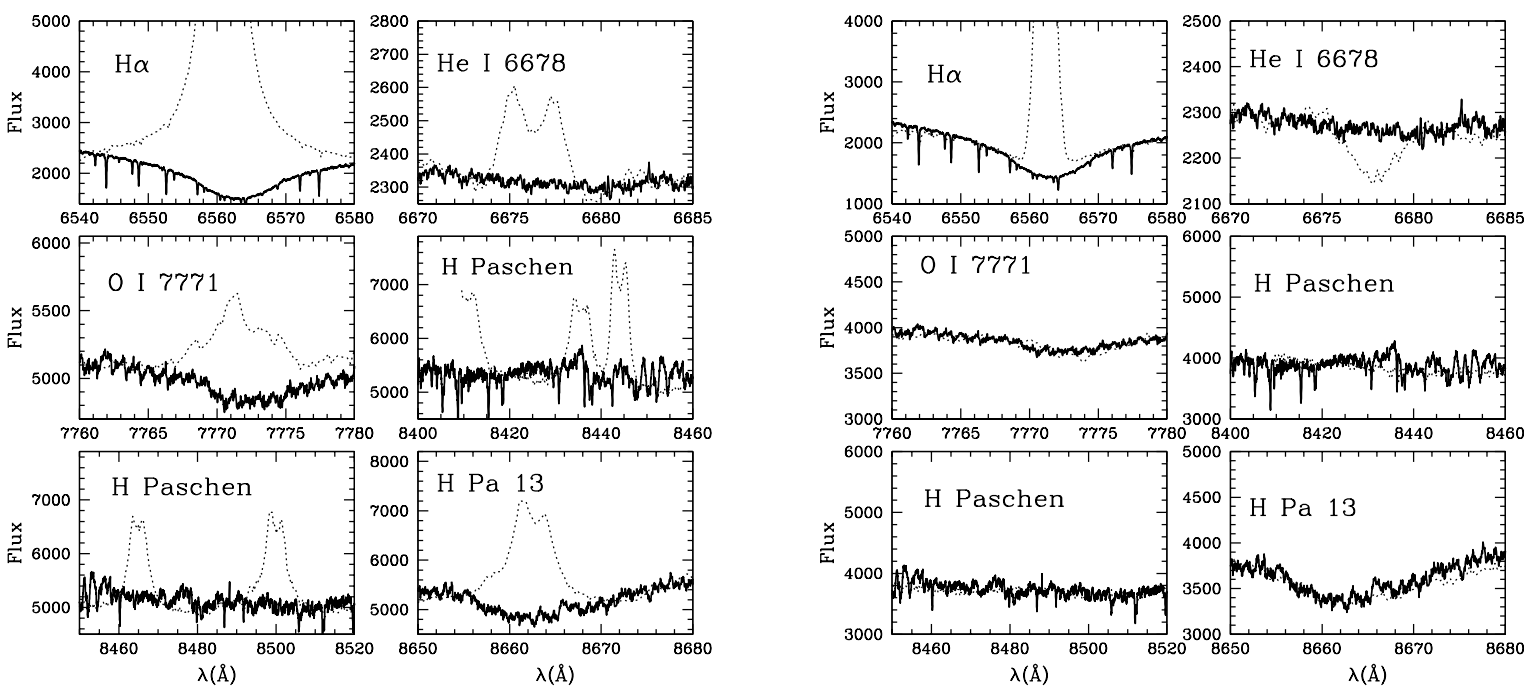

Fig. 7.- Comparison of the optical spectrum of $\eta$ Cha (thick line) with the classical Be star KUN314s (left, dotted line) and the B7 star with a transition/debris disk MVA 437 (right, dotted line), both young ( $\sim 4$ Myr) stars in Tr 37 (Sicilia-Aguilar et al. 2007). The spectra have been scaled to match the continuum levels. The $\eta$ Cha spectrum is fully consistent with a normal young B star, with some variations due to rotation, accretion/activity (MVA 437 presents $\mathrm{H} \alpha$ emission due to either chromospheric activity or very low accretion, while $\eta$ Cha does not) and spectral type. The flux is given in arbitrary units. 

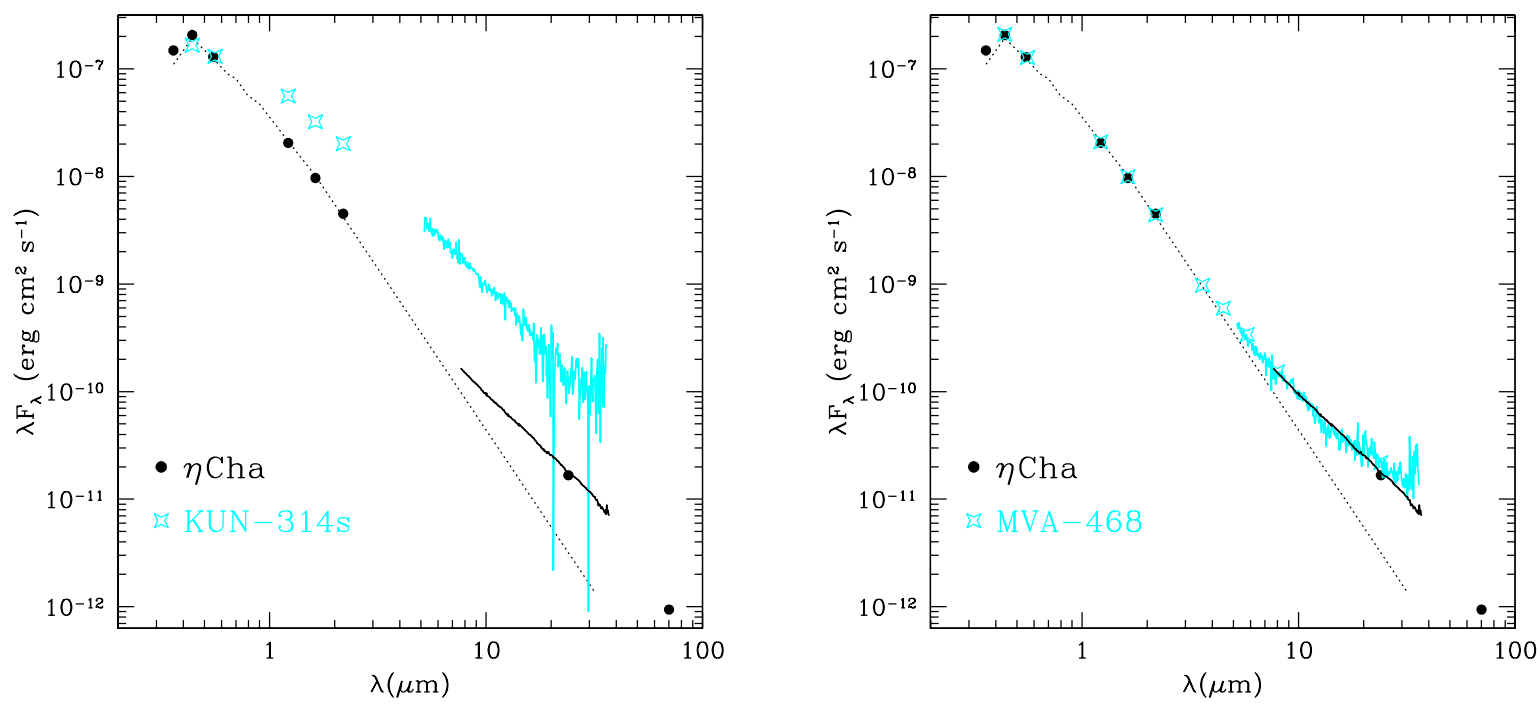

Fig. 8.- Comparison of the SEDs of $\eta$ Cha with the classical Be star KUN-314s (left) and the B7 star with a transition/debris disk MVA 468 (right; Sicilia-Aguilar et al. 2007). The SEDs of KUN-314s and MVA-468 have been scaled to their optical and JHK fluxes to match the $\eta$ Cha distance. Although the slope of the $\eta$ Cha disk is roughly consistent with that of a classical Be star, as it lacks features, those characteristics are not unique of classical Be stars, being present in some transitional and/or young debris disks. 
Table 1. Stellar parameters

\begin{tabular}{|c|c|c|c|c|c|c|c|}
\hline Name & Sp. Type & $\mathrm{H} \alpha \mathrm{EW}(\AA)$ & $\dot{\mathrm{M}}\left(10^{-8} \mathrm{M}_{\odot \mathrm{yr}^{-1}}\right)$ & Н $\alpha$ Type & SED Type & Binary & References \\
\hline$\eta$ Cha & B8+M1: ${ }^{a}$ & 8.5 & & & Class III/debris & & 1 \\
\hline RS Cha & $\mathrm{A} 7+\mathrm{A} 8+\mathrm{M} 1:^{a}$ & 9.2 & & & Class III & eclipsing A7/A8 & 1 \\
\hline HD 75505 & A5 & & & & Class III & & 2 \\
\hline J0836 & M5.3+M5.3: & & & & Class III & $<0.04$ & 3 \\
\hline J0838 & M5.0+M5.0: & & & & Class III & $<0.04$ & 3 \\
\hline J0841 & M4.7 & -12.0 & & WTTS & TO/flat & & 3,4 \\
\hline J0843 & M3.4 & -90 & 0.1 & CTTS & Class II & & 3,5 \\
\hline J0844 & M5.5 & & & & flat & & 3 \\
\hline RECX-1 & $\mathrm{K} 7.0+\mathrm{M} 0.0$ & -1.4 & & WTTS & Class III & 0.1 & $3,1,6$ \\
\hline RECX-3 & M3.0 & -2.2 & & WTTS & TO & & 3,5 \\
\hline RECX-4 & M1.3 & -2.3 & & WTTS & TO & & 3,5 \\
\hline RECX-5 & M3.8 & -35 & 0.005 & CTTS & TO & & 3,5 \\
\hline RECX-6 & M3.0 & -3.6 & & WTTS & Class III & & 3,5 \\
\hline RECX-7 & K6.9+M1.0 & -0.4 & & WTTS & Class III & 0.001 & $3,5,7,8$ \\
\hline RECX-9 & $\mathrm{M} 4.4+\mathrm{M} 4.7$ & -10 & 0.004 & CTTS & TO & 0.2 & $3,5,6$ \\
\hline RECX-10 & M0.3 & -1.0 & & WTTS & Class III & & 3,5 \\
\hline RECX-11 & K6.5 & -3.2 & 0.004 & CTTS & Class II & & 3,5 \\
\hline RECX-12 & M3.2+M3.2: & -4.2 & & WTTS & Class III & 0.04 & $1,3,5,9$ \\
\hline
\end{tabular}

Note. - Uncertain values are marked with ':'. The SEDs are classified as Class II (comparable to Taurus), flattened disks $\left(\lambda \mathrm{F}_{\lambda} \sim \lambda^{-4 / 3}\right)$, TO (zero or very small near-IR excess), and Class III (no IR excess over the stellar photosphere), see text for more details. $\eta$ Cha is defined as a Class III source with a second generation "debris" disk. The "binary" column indicates the projected separation (in arcsec), when known, the spectral types of the two components are listed in the corresponding column. ${ }^{a}$ : The low-mass companions to $\eta$ Cha and RS Cha are speculative, based on the X-ray emission from these stars having roughly the properties of a roughly 0.5 solar mass T Tauri star (spectral type $\sim \mathrm{M} 1$ ). References for the spectral types, H $\alpha$, and accretion status: $1=$ Mamajek et al. (1999); $2=$ Lawson et al. (2001); $3=$ Lyo et al. (2004b); $4=$ Lawson et al. (2002); $5=$ Lawson et al. (2004); $6=$ Köhler \& Petr-Gotzens (2002); $7=$ Moraux et al. (2007); $8=$ Lyo et al. (2003); $9=$ Brandecker et al. (2006). Note that for the H $\alpha$ class, we give priority to the detection of broad $\mathrm{H} \alpha$ lines (sign of accretion) to classify an object as a CTTS, rather than to the $\mathrm{H} \alpha \mathrm{EW}$ alone, that may be misleading in cases of very low mass accretion rates (Sicilia-Aguilar et al. 2006b). 
Table 2. Optical photometry from the literature

\begin{tabular}{lccccccc}
\hline \hline \multicolumn{1}{c}{ Name } & 2 MASS ID & U-B & B-V & V & V-R $_{c}$ & V-I $_{c}$ & Refs. \\
\hline$\eta$ Cha & $08411947-7857481$ & -0.35 & -0.10 & 5.47 & - & - & 1,2 \\
RS Cha & $08431222-7904123$ & 0.07 & 0.24 & 6.04 & - & - & 2,3 \\
HD-75505 & $08414471-7902531$ & - & - & 7.27 & 0.06 & 0.15 & $2,5,6$ \\
J0838 & $08385150-7916136$ & - & - & 16.82 & 1.61 & 3.54 & 7,2 \\
J0841 & $08413030-7853064$ & - & - & 17.07 & 1.52 & 3.25 & 7,2 \\
J0843 & $08431857-7905181$ & - & - & 13.97 & 0.99 & 2.20 & 2,7 \\
J0844 & $08440914-7833457$ & - & 0.49 & 17.74 & - & - & 2,8 \\
J0836 & $08361072-7908183$ & - & - & 17.66 & 1.72 & 3.76 & 2,6 \\
RECX-1 & $08365623-7856454$ & - & 1.23 & 10.61 & 0.71 & 1.42 & 2,6 \\
RECX-3 & $08413703-7903304$ & - & 1.51 & 14.37 & 1.16 & 2.58 & $2,4,5$ \\
RECX-4 & $08422372-7904030$ & - & 1.45 & 12.73 & 0.93 & 1.92 & $2,4,5$ \\
RECX-5 & $08422710-7857479$ & - & 1.51 & 15.20 & 1.29 & 2.81 & $2,4,5$ \\
RECX-6 & $08423879-7854427$ & - & 1.50 & 14.08 & 1.00 & 2.40 & $2,4,5$ \\
RECX-7 & $08430723-7904524$ & - & 1.19 & 10.84 & 0.67 & 1.40 & $2,4,5$ \\
RECX-9 & $08441637-7859080$ & - & 1.52 & 15.00 & 1.36 & 2.99 & $2,4,5$ \\
RECX-10 & $08443188-7846311$ & - & 1.45 & 12.53 & 0.86 & 1.78 & $2,4,5$ \\
RECX-11 & $08470165-7859345$ & - & 1.22 & 11.13 & 0.66 & 1.37 & $2,4,5$ \\
RECX-12 & $08475676-7854532$ & - & 1.51 & 13.17 & 1.07 & 2.37 & $2,4,5$ \\
\hline
\end{tabular}

Note. - Photometry observations of the $\eta$ Cha members. References: $1=$ Johnson et al. (1966); $2=2$ MASS, Cutri et al. (2003); $3=$ Mermilliod (1991); $4=$ Lawson et al. (2001); $5=$ Lyo et al. (2004); $6=$ Lyo et al. (2003); $7=$ Lawson et al. (2002); 8 = NOMAD Catalog, Zacharias et al. (2005), provided by Vizier. 
Table 3. IR photometry

\begin{tabular}{|c|c|c|c|c|c|c|c|c|c|c|}
\hline Name & 2MASS ID & $\mathrm{J}$ & $\mathrm{H}$ & $\mathrm{K}$ & $3.6 \mu \mathrm{m}$ & $4.5 \mu \mathrm{m}$ & $5.8 \mu \mathrm{m}$ & $8.0 \mu \mathrm{m}$ & $24 \mu \mathrm{m}$ & $70 \mu \mathrm{m}$ \\
\hline$\eta$-Cha & $08411947-7857481$ & $5.69 \pm 0.02$ & $5.72 \pm 0.04$ & $5.72 \pm 0.02$ & $a$ & $a$ & $a$ & $a$ & $4.33 \pm 0.02$ & $3.87 \pm 0.13:$ \\
\hline RS-Cha & 08431222-7904123 & $5.99 \pm 0.03$ & $5.88 \pm 0.04$ & $5.85 \pm 0.03$ & $a$ & $a$ & $5.40 \pm 0.01$ & $5.40 \pm 0.01$ & $5.34 \pm 0.03$ & - \\
\hline HD-75505 & 08414471-7902531 & $7.06 \pm 0.03$ & $6.99 \pm 0.04$ & $6.93 \pm 0.02$ & $6.97 \pm 0.01$ & $6.97 \pm 0.01$ & $6.97 \pm 0.01$ & $6.97 \pm 0.01$ & $6.97 \pm 0.06$ & - \\
\hline J0836 & $08361072-7908183$ & $11.85 \pm 0.02$ & $11.28 \pm 0.03$ & $10.95 \pm 0.02$ & $10.57 \pm 0.03$ & $10.45 \pm 0.01$ & $10.40 \pm 0.01$ & $10.37 \pm 0.01$ & $b$ & $b$ \\
\hline J0838 & 08385150-7916136 & $11.28 \pm 0.02$ & $10.72 \pm 0.02$ & $10.43 \pm 0.02$ & $10.07 \pm 0.02$ & $10.00 \pm 0.01$ & $9.92 \pm 0.01$ & $9.90 \pm 0.01$ & $b$ & $b$ \\
\hline J0841 & 08413030-7853064 & $11.81 \pm 0.03$ & $11.24 \pm 0.03$ & $10.98 \pm 0.02$ & $10.53 \pm 0.01$ & $10.26 \pm 0.05$ & $10.04 \pm 0.01$ & $9.48 \pm 0.01$ & $7.07 \pm 0.06$ & - \\
\hline J0843 & $08431742-7905236$ & $13.53 \pm 0.05$ & $13.18 \pm 0.08$ & $13.09 \pm 0.04$ & $8.38 \pm 0.03$ & $7.91 \pm 0.01$ & $7.42 \pm 0.03$ & $6.51 \pm 0.01$ & $3.52 \pm 0.01$ & $1.89 \pm 0.02$ \\
\hline J0844 & 08440914-7833457 & $12.51 \pm 0.02$ & $11.98 \pm 0.02$ & $11.62 \pm 0.02$ & $11.02 \pm 0.01$ & $10.75 \pm 0.01$ & $10.42 \pm 0.01$ & $9.76 \pm 0.01$ & $7.11 \pm 0.07$ & $b$ \\
\hline RECX-1 & 08365623-7856454 & $8.15 \pm 0.02$ & $7.50 \pm 0.05$ & $7.34 \pm 0.02$ & $7.17 \pm 0.02$ & $7.20 \pm 0.02$ & $7.16 \pm 0.01$ & $7.11 \pm 0.02$ & $6.96 \pm 0.06$ & - \\
\hline RECX-3 & 08413703-7903304 & $10.35 \pm 0.02$ & $9.65 \pm 0.02$ & $9.41 \pm 0.02$ & $9.27 \pm 0.02$ & $9.21 \pm 0.02$ & $9.09 \pm 0.05$ & $9.15 \pm 0.01$ & $8.49 \pm 0.13$ & - \\
\hline RECX-4 & 08422372-7904030 & $9.54 \pm 0.02$ & $8.78 \pm 0.06$ & $8.62 \pm 0.02$ & $8.45 \pm 0.02$ & $8.45 \pm 0.02$ & $8.37 \pm 0.01$ & $8.32 \pm 0.02$ & $7.73 \pm 0.09$ & - \\
\hline RECX-5 & 08422710-7857479 & $10.78 \pm 0.02$ & $10.10 \pm 0.02$ & $9.86 \pm 0.02$ & $9.59 \pm 0.03$ & $9.50 \pm 0.03$ & $9.37 \pm 0.01$ & $8.89 \pm 0.01$ & $5.05 \pm 0.02$ & $2.38 \pm 0.04$ \\
\hline RECX-6 & 08423879-7854427 & $10.23 \pm 0.03$ & $9.58 \pm 0.02$ & $9.29 \pm 0.02$ & $9.15 \pm 0.04$ & $9.07 \pm 0.07$ & $9.04 \pm 0.01$ & $9.04 \pm 0.01$ & $8.88 \pm 0.16$ & - \\
\hline RECX-7 & 08430723-7904524 & $8.42 \pm 0.02$ & $7.76 \pm 0.03$ & $7.63 \pm 0.03$ & $7.52 \pm 0.01$ & $7.54 \pm 0.02$ & $7.49 \pm 0.01$ & $7.46 \pm 0.01$ & $7.37 \pm 0.08$ & - \\
\hline RECX-9 & 08441637-7859080 & $10.26 \pm 0.03$ & $9.67 \pm 0.03$ & $9.34 \pm 0.02$ & $8.99 \pm 0.02$ & $8.80 \pm 0.01$ & $8.57 \pm 0.01$ & $7.97 \pm 0.02$ & $5.38 \pm 0.03$ & $3.16 \pm 0.07$ \\
\hline RECX-10 & 08443188-7846311 & $9.65 \pm 0.02$ & $8.92 \pm 0.06$ & $8.73 \pm 0.02$ & $8.58 \pm 0.01$ & $8.61 \pm 0.01$ & $8.55 \pm 0.02$ & $8.49 \pm 0.01$ & $8.45 \pm 0.13$ & - \\
\hline RECX-11 & 08470165-7859345 & $8.73 \pm 0.02$ & $8.03 \pm 0.06$ & $7.66 \pm 0.04$ & $7.09 \pm 0.02$ & $6.86 \pm 0.01$ & $6.57 \pm 0.01$ & $5.97 \pm 0.01$ & $3.68 \pm 0.01$ & $1.80 \pm 0.02$ \\
\hline RECX-12 & 08475676-7854532 & $9.32 \pm 0.02$ & $8.68 \pm 0.08$ & $8.41 \pm 0.03$ & $8.19 \pm 0.01$ & $8.15 \pm 0.01$ & $8.10 \pm 0.02$ & $8.07 \pm 0.01$ & $7.95 \pm 0.10$ & - \\
\hline
\end{tabular}

Note. - IR Photometry observations of the $\eta$ Cha members. ${ }^{a}=$ The object is saturated. ${ }^{b}=$ The object is out of the MIPS field. The JHK data is obtained from 2MASS (Cutri et al. 2003), and the IRAC data was published in Megeath et al. (2005). The MIPS magnitudes have an extra error around 10\% due to conversion factors and different chip coverage. Uncertain values are marked with ' $:$ '. 
Table 4. Silicate Composition in $\eta$ Cha (TLTD)

\begin{tabular}{|c|c|c|c|c|c|}
\hline Name & $\begin{array}{l}0.1 \mu \mathrm{m} \text { A.Ol. } \\
1.5 \mu \mathrm{m} \mathrm{A.Ol} . \\
6.0 \mu \mathrm{m} \text { A.Ol. }\end{array}$ & $\begin{array}{l}0.1 \mu \mathrm{m} \text { A.Py. } \\
1.5 \mu \mathrm{m} \text { A.Py. } \\
6.0 \mu \mathrm{m} \text { A.Py. }\end{array}$ & $\begin{array}{l}0.1 \mu \mathrm{m} \text { Fors. } \\
1.5 \mu \mathrm{m} \text { Fors. } \\
6.0 \mu \mathrm{m} \text { Fors. }\end{array}$ & $\begin{array}{c}0.1 \mu \mathrm{m} \text { Enst. } \\
1.5 \mu \mathrm{m} \text { Enst. } \\
-\end{array}$ & $\begin{array}{l}0.1 \mu \mathrm{m} \text { Sil. } \\
1.5 \mu \mathrm{m} \text { Sil. } \\
6.0 \mu \mathrm{m} \text { Sil. }\end{array}$ \\
\hline \multirow[t]{3}{*}{ J0843 } & - & - & $11.5_{-0.6}^{+0.6}$ & $5.5_{-1.2}^{+1.2}$ & $2.8_{-0.4}^{+0.3}$ \\
\hline & - & $68_{-3}^{+3}$ & $4_{-2}^{+2}$ & $8_{-2}^{+-.2}$ & -0.4 \\
\hline & - & - & -2 & - & - \\
\hline \multirow[t]{3}{*}{ J0844 } & $17_{-13}^{+19}$ & $60_{-13}^{+13}$ & $<3$ & $<8$ & - \\
\hline & - & $<24$ & $4_{-4}^{+11}$ & $15_{-10}^{+13}$ & $<5$ \\
\hline & $<4$ & - & $<31$ & - & - \\
\hline \multirow[t]{3}{*}{ RECX-5 } & - & - & $10.0_{-0.5}^{+0.6}$ & - & - \\
\hline & $38_{-8}^{+9}$ & $37_{-10}^{+10}$ & - & $12_{-2}^{+2}$ & $2.0_{-0.8}^{+0.9}$ \\
\hline & - & - & - & - & - \\
\hline \multirow[t]{3}{*}{ RECX-9 } & - & $20_{-12}^{+22}$ & $6_{-1}^{+1}$ & $<3$ & $2.7_{-0.9}^{+0.8}$ \\
\hline & - & $57_{-26}^{+14}$ & $<3$ & $13_{-5}^{+5}$ & - \\
\hline & $<7$ & - & $<9$ & - & - \\
\hline \multirow[t]{3}{*}{ RECX-11 } & - & - & - & - & - \\
\hline & - & $12_{-1}^{+1}$ & - & $3.7_{-0.5}^{+0.4}$ & - \\
\hline & $74_{-3}^{+2}$ & - & - & - & $7_{-2}^{+2}$ \\
\hline \multirow[t]{3}{*}{$\mathrm{J} 0843^{l}$} & $4_{-3}^{+4}$ & - & $2.7_{-0.2}^{+0.1}$ & - & - \\
\hline & $89_{-4}^{+3}$ & - & - & $3.0_{-0.1}^{+0.1}$ & - \\
\hline & - & - & - & - & - \\
\hline \multirow[t]{3}{*}{ RECX-5l } & - & - & $7.6_{-1.3}^{+1.1}$ & - & - \\
\hline & $52_{-9}^{+5}$ & $32_{-6}^{+5}$ & - & - & - \\
\hline & - & $6_{-5}^{+22}$ & - & - & $<3$ \\
\hline \multirow[t]{3}{*}{ RECX-11 ${ }^{l}$} & - & - & $3.7_{-0.5}^{+0.6}$ & $<3$ & - \\
\hline & $19_{-11}^{+8}$ & $63_{-8}^{+8}$ & $3_{-1}^{+1}$ & - & $3.3_{-0.5}^{+0.5}$ \\
\hline & $<2$ & - & $4_{-3}^{+3}$ & - & $<6$ \\
\hline \multirow[t]{3}{*}{ RECX-9 $9^{l}$} & - & $4_{-4}^{+54}$ & $3_{-2}^{+3}$ & $<6$ & $5_{-4}^{+3}$ \\
\hline & $<27$ & $70_{-26}^{+13}$ & $7_{-4}^{+6}$ & $3_{-2}^{+3}$ & - \\
\hline & $<28$ & $3_{-3}^{+34}$ & $2_{-2}^{+11}$ & - & $<7$ \\
\hline
\end{tabular}


Note. - Silicate fit for the objects in $\eta$ Cha using the Two Layer Temperature Distribution method (J09). The values are percentages in mass fraction. If no value is given for a certain species/size, it means that it is present at $2 \%$ level or less. The silicate compositions corresponding to the $7-17 \mu \mathrm{m}$ range fit are given in the first part of the table, followed by the $17-35 \mu \mathrm{m}$ fit, which are also marked with ${ }^{l}$ added to the name. 
Table 5. Temperatures in the TLTD fit

\begin{tabular}{lccc}
\hline \hline \multicolumn{1}{c}{ Name } & $\mathrm{T}_{\text {rim }}(\mathrm{K})$ & $\mathrm{T}_{\text {mid }}(\mathrm{K})$ & $\mathrm{T}_{a t m}(\mathrm{~K})$ \\
\hline J0843 & $162-1590$ & $101-209$ & $116-1395$ \\
J0844 & $156-1578$ & $116-293$ & $116-1389$ \\
RECX-5 & $159-1564$ & $115-178$ & $116-362$ \\
RECX-9 & $156-1586$ & $114-324$ & $115-934$ \\
RECX-11 & $163-1598$ & $116-218$ & $116-963$ \\
& & & \\
& & & \\
J0843 $^{l}$ & $163-1562$ & $59-118$ & $60-321$ \\
RECX-5 $^{l}$ & $159-1590$ & $106-145$ & $116-174$ \\
RECX-9 $^{l}$ & $159-1595$ & $115-189$ & $115-221$ \\
RECX-11 $^{l}$ & $163-1598$ & $116-196$ & $115-235$ \\
\hline
\end{tabular}

Note. - Maximum and minimum temperatures (in $\mathrm{K}$ ) of the three components fitted by the TLTD model (inner rim, disk midplane, and disk atmosphere). See text for details. 
Table 6. Grain sizes and crystalline fraction (TLTD)

\begin{tabular}{lccc}
\hline \hline \multicolumn{1}{c}{ Name } & Size (am.) $/ \mu \mathrm{m}$ & Size (cryst.) $/ \mu \mathrm{m}$ & Cryst. \\
\hline J0843 & $1.5_{-0.1}^{+0.1}$ & $0.7_{-0.1}^{+0.1}$ & $29_{-2}^{+2}$ \\
J0844 & $0.1_{-0.1}^{+0.3}:$ & $<6:$ & $21_{-9}^{+9}:$ \\
RECX-5 & $1.5_{-0.1}^{+0.1}$ & $0.9_{-0.1}^{+0.1}$ & $22_{-2}^{+2}$ \\
RECX-9 & $1.2_{-0.3}^{+0.2}$ & $1.0_{-0.2}^{+0.1}$ & $19_{-3}^{+5}$ \\
RECX-11 & $3.4_{-0.1}^{+0.1}$ & $0.9_{-0.1}^{+0.1}$ & $6.5_{-0.5}^{+0.4}$ \\
& & & \\
J0843 $l$ & & & \\
RECX-5 $l$ & $1.4_{-0.1}^{+0.1}$ & $0.8_{-0.1}^{+0.1}$ & $5.8_{-0.2}^{+0.1}$ \\
RECX-9 ${ }^{l}$ & $1.8_{-0.3}^{+0.9}$ & $0.2_{-0.1}^{+0.1}$ & $9_{-1}^{+1}$ \\
RECX-11 $^{l}$ & $2_{-1}^{+1}:$ & $1.2_{-0.4}^{+0.6}:$ & $16_{-7}^{+9}:$ \\
\hline
\end{tabular}

Note. - Average grain sizes for amorphous and crystalline silicates and crystalline fraction (mass fraction of crystalline silicates) from the TLTD fit. The results corresponding to the $7-17 \mu \mathrm{m}$ range fit are given in the first part of the table, followed by the 17-35 $\mu \mathrm{m}$ fit, which are also marked with ${ }^{l}$ added to the name. The colon (:) indicates uncertain values in spectra with poor $\mathrm{S} / \mathrm{N}$. 